\title{
CERTAIN HYPERGEOMETRIC SERIES RELATED TO THE ROOT SYSTEM $B C$
}

\author{
R. J. BEERENDS AND E. M. OPDAM
}

\begin{abstract}
We show that the generalized hypergeometric function ${ }_{2} F_{1}$ of matrix argument is the series expansion at the origin of a special case of the hypergeometric function associated with the root system of type $B C$. In addition we prove that the Jacobi polynomials of matrix argument correspond to the Jacobi polynomials associated with the root system of type $B C$. We also give a precise relation between Jack polynomials and the Jacobi polynomials associated with the root system of type $A$. As a side result one obtains generalized hooklength formulas which are related to Harish-Chandra's c-function and one can prove a conjecture due to Macdonald relating two inner products on a space of symmetric functions.
\end{abstract}

\section{INTRODUCTION}

Various extensions of the classical Gauss hypergeometric function to several variables exist in the literature. In this paper we will unify two different approaches to hypergeometric functions in several variables and the related Jacobi polynomials. We will show that the generalized hypergeometric function of matrix argument is a special case of the hypergeometric function associated with root systems. A similar result holds for the generalized Jacobi polynomials of matrix argument.

One of the generalizations of the classical one-variable hypergeometric function ${ }_{p} F_{q}$ is the hypergeometric function ${ }_{p} F_{q}$ of matrix argument which was introduced in 1955 by Herz [8] using Laplace and inverse Laplace transforms. This idea originated in a paper by Bochner who considered Bessel functions of matrix argument (see [8] for details). Constantine [2] then found a series expansion for the ${ }_{p} F_{q}$ 's in terms of so-called zonal polynomials. These zonal polynomials are the spherical functions of certain irreducible polynomial representations of $G L(n, \mathbf{R})$. The zonal polynomials and the hypergeometric functions were studied extensively by Constantine, James and Muirhead (cf. [2, 9, $10,26]$ and the references given there). In $[9, \S 8]$ the analogous situation for $G L(n, \mathrm{C})$ is treated and the complex zonal polynomials occur. Recently Gross and Richards [5] treated the cases $G L(n, \mathbf{F})(\mathbf{F}=\mathbf{R}, \mathbf{C}$ or $\mathbf{H})$ simultaneously.

Received by the editors June 4, 1991.

1991 Mathematics Subject Classification. Primary 33C80; Secondary 22E30, 33C50, 33C70, $42 \mathrm{C} 05$.

Research of both authors was supported partially by a grant from the Netherlands organization for scientific research (NWO). 
If we now put $k=\frac{1}{2} \operatorname{dim}_{\mathbf{R}} \mathbf{F}$ then we can consider $k$ as a parameter in the definition of ${ }_{p} F_{q}$ in [5, 6.1(1)]. Faraut and Korányi associate in [4] hypergeometric functions ${ }_{p} F_{q}$ with any so-called symmetric cone and in particular they obtain the cases $k=\frac{1}{2}, 1$ and 2 above. Hence they extended the number of values for which one can define these ${ }_{p} F_{q}$ 's. From the definition [5, 6.1(1)] or [4, (2.4)] it is clear how to generalize the definition of the ${ }_{p} F_{q}$ 's to arbitrary values of $k$, except for the generalization of the zonal polynomials. Zonal polynomials however are closely related to the Jacobi polynomials associated with $S L(n, \mathbf{F})$, i.e. with root system $A_{n-1}$; these Jacobi polynomials have been generalized to arbitrary $k$ by Heckman and Opdam in [6 and 7]. By now a different approach to the generalization of the zonal polynomials is well established in the literature. This approach is due to Macdonald who noted that the so-called Jack polynomials are in fact a generalization to arbitrary $k$ of the zonal polynomials (cf. $[20,21,22,30])$. The generalized hypergeometric functions ${ }_{p} F_{q}$ of matrix argument were then defined independently by Koranyi [15] and Macdonald [24] in terms of the Jack polynomials instead of the Jacobi polynomials for $A_{n-1}$. Let us note here that this paper deals exclusively with the case $p=2, q=1$.

The classical Gauss hypergeometric function ${ }_{2} F_{1}$ can be interpreted in terms of the rank-one root system of type $B C$. Heckman and Opdam $[6,7,27]$ associate with any higher rank root system a ${ }_{2} F_{1}$ hypergeometric function, thus giving a completely different multivariable generalization of the classical ${ }_{2} F_{1}$. We will show that the generalized ${ }_{2} F_{1}$ of matrix argument is in fact the series expansion "at the origin" of a special case of the hypergeometric function associated with $B C_{n}$. The two variable case has essentially been established by Yan $[32,33]$. In addition we will prove that the Jacobi polynomials as defined by Herz [8], James and Constantine [10] and Macdonald [24] correspond to the multivariable Jacobi polynomials associated with $B C_{n}$ as defined in [6 and 7].

The paper is organized as follows. After some notations and preliminaries we show in $\S 3$ how the Jacobi polynomials associated with $A_{n-1}$ are related to the Jack polynomials. This result also led us to lower and upper hook-length formulas which generalize the classical (i.e. $k=1$ ) hook-length formula. As an application we prove a conjecture due to Macdonald [21, §4] relating two inner products on a space of symmetric functions; this conjecture also follows from the material in [20, Chapter VI]. In $\S 4$ we prove how the generalized hypergeometric function ${ }_{2} F_{1}$ of matrix argument can be obtained as a special case of the hypergeometric function associated with $B C_{n}$. Finally we treat the Jacobi polynomials associated with $B C_{n}$ in $\S 5$.

\section{NOTATIONS AND PRELIMINARIES}

a. Jack polynomials and the generalized hypergeometric function of matrix argument. For unexplained results concerning partitions and symmetric functions we refer to [19, Chapter I]. A partition $\lambda$ is any sequence $\lambda=\left(\lambda_{1}, \lambda_{2}, \ldots, \lambda_{n}\right)$ of nonnegative integers such that $\lambda_{1} \geq \lambda_{2} \geq \cdots \geq \lambda_{n} \geq 0$. The number of $\lambda_{i} \neq 0$ is called the length of $\lambda$ and is denoted by $l(\lambda)$. The weight $|\lambda|$ of $\lambda$ is defined as

$$
|\lambda|=\sum_{i \geq 1} \lambda_{i} .
$$

Given a partition $\lambda=\left(\lambda_{1}, \lambda_{2}, \ldots, \lambda_{n}\right)$ we define the dual partition $\lambda^{\prime}=$ 
$\left(\lambda_{1}^{\prime}, \lambda_{2}^{\prime}, \ldots, \lambda_{m}^{\prime}\right)$ by $\lambda_{i}^{\prime}=\operatorname{Card}\left\{j \mid \lambda_{j} \geq i\right\}$. Furthermore we put

$$
n(\lambda)=\sum_{i \geq 1}(i-1) \lambda_{i}=\sum_{i \geq 1}\left(\begin{array}{c}
\lambda_{i}^{\prime} \\
2
\end{array}\right)
$$

The diagram of a partition $\lambda$ is the set of points $(i, j) \in \mathbf{Z}^{2}$ such that $1 \leq j \leq$ $\lambda_{i}$; we will simply write $(i, j) \in \lambda$ if $(i, j)$ belongs to the diagram of $\lambda$. We write $\mu \leq \lambda$ if $|\mu|=|\lambda|$ and $\sum_{i=1}^{k} \mu_{i} \leq \sum_{i=1}^{k} \lambda_{i}$ for all $k \geq 1$. This is a partial ordering on the set of partitions of a given weight. We write $\mu \subset \lambda$ if $\mu_{i} \leq \lambda_{i}$ for all $i$.

Denote by $\Lambda_{n}$ the ring of symmetric polynomials in $n$ independent variables with integer coefficients. If $\alpha=\left(\alpha_{1}, \alpha_{2}, \ldots, \alpha_{n}\right) \in\left(\mathbf{Z}^{+}\right)^{n}\left(\mathbf{Z}^{+}\right.$the set of nonnegative integers) then we put $|\alpha|=\alpha_{1}+\alpha_{2}+\cdots+\alpha_{n}$ and we let $x^{\alpha}$ denote the monomial $x_{1}^{\alpha_{1}} x_{2}^{\alpha_{2}} \cdots x_{n}^{\alpha_{n}}$. For a partition $\lambda$ of length $l(\lambda) \leq n$ we define the monomial symmetric polynomial $m_{\lambda}$ in $\Lambda_{n}$ by

$$
m_{\lambda}=m_{\lambda}\left(x_{1}, x_{2}, \ldots, x_{n}\right)=\sum x^{\alpha},
$$

where the sum is taken over all distinct permutations $\alpha$ of $\lambda$. If $l(\lambda)>n$ we put $m_{\lambda}=0$. The power sums $p_{r}$ are defined for each integer $r \geq 1$ by $p_{r}=\sum_{i=1}^{n} x_{i}^{r}=m_{(r)}$. For each partition $\lambda=\left(\lambda_{1}, \lambda_{2}, \ldots, \lambda_{n}\right)$ define $p_{\lambda}=$ $p_{\lambda_{1}} p_{\lambda_{2}} \cdots p_{\lambda_{n}}$. Finally, $s_{\lambda}$ will denote the Schur function corresponding to the partition $\lambda$ (cf. [19, I.3]). It is well known that the sets $\left(m_{\lambda}\right)$ and $\left(s_{\lambda}\right)$ form a Z-basis for $\Lambda_{n}$.

We now follow Stanley's introduction of the Jack polynomials [30, Chapter I]. For a partition $\lambda$ we write

$$
z_{\lambda}=\prod_{i \geq 1} i^{m_{i}} m_{i} !
$$

where $m_{i}$ is the number of parts of $\lambda$ equal to $i$. Let $k$ be a parameter and $\mathbf{Q}(k)$ the field of all rational functions of $k$ with coefficients in $\mathbf{Q}$. For convenience we will work temporarily with symmetric functions in infinitely many variables. In particular we let $m_{\lambda}$ and $p_{\lambda}$ denote the symmetric functions in infinitely many variables in the ring of symmetric functions $\Lambda$ as defined in $[19, \S 2]$. The $m_{\lambda}$ form a Z-basis of $\Lambda$ and the $p_{\lambda}$ form a $\mathbf{Q}$-basis of $\Lambda \otimes \mathbf{Q}$, the ring of symmetric functions with coefficients in $\mathbf{Q}$. Let $\Lambda \otimes \mathbf{Q}(k)$ denote the ring of symmetric functions with coefficients in $\mathbf{Q}(k)$ and define a scalar product on $\Lambda \otimes \mathbf{Q}(k)$ by the condition

$$
\left\langle p_{\lambda}, p_{\mu}\right\rangle_{k}=\delta_{\lambda \mu} z_{\lambda} k^{-l(\lambda)},
$$

where $\delta_{\lambda \mu}$ is the Kronecker delta. We quote the following theorem from [30, Chapter I].

Theorem 2.1. There are unique symmetric functions $J_{\lambda}=J_{\lambda}\left(x ; k^{-1}\right)$ in $\Lambda \otimes$ $\mathbf{Q}(k)$ indexed by partitions $\lambda$, such that

1. $\left\langle J_{\lambda}, J_{\mu}\right\rangle_{k}=0$ if $\lambda \neq \mu$;

2. if

$$
J_{\lambda}\left(x ; k^{-1}\right)=\sum_{\mu} v_{\lambda \mu}\left(k^{-1}\right) m_{\mu}(x)
$$

then $v_{\lambda \mu}\left(k^{-1}\right)=0$ unless $\mu \leq \lambda$; 
3. If $|\lambda|=l$ then the coefficient $v_{\lambda,\left(1^{l}\right)}$ of $x_{1} x_{2} \cdots x_{l}$ in $J_{\lambda}$ equals $l$ !.

If we set $x_{n+1}=x_{n+2}=\cdots=0$ in $J_{\lambda}$ then we obtain for any partition of length $l(\lambda) \leq n$ a symmetric polynomial $J_{\lambda}\left(x_{1}, \ldots, x_{n} ; k^{-1}\right)$ in $\Lambda_{n} \otimes \mathbf{Q}(k)$, homogeneous of degree $|\lambda|$. The $J_{\lambda}\left(x_{1}, \ldots, x_{n} ; k^{-1}\right)$ vanish for $l(\lambda)>n$ and are linearly independent otherwise [30, Proposition 2.5$]$. We will call these symmetric polynomials the Jack polynomials. Throughout this paper $n$ will be fixed.

Remark 1. Theorem 2.1 is a special case of a more general existence theorem due to Macdonald [22, Theorem 2.3 and also 20, Chapter VI, §4]. Since we will only need the case of the Jack polynomial, we refer to [30], where many more details and results on Jack polynomials can be found. For a different approach to Jack polynomials also see [21].

Remark 2. In [30, 20, Chapter VI, 21, 22, $\S 1.7$ and 24] the parameter $\alpha=k^{-1}$ is used. We will use $\alpha$ differently later on. To avoid confusion we prefer to write $J_{\lambda}\left(x ; k^{-1}\right)$ instead of $J_{\lambda}(x ; k)$. This explains the rather awkward notation.

Remark 3. The Jack polynomials reduce to the so-called zonal polynomials $C_{\lambda}$ if $k=\frac{1}{2}$, although the $C_{\lambda}$ in e.g. [2] are normalized differently. In fact $C_{\lambda}(x)=2^{|\lambda|}|\lambda| ! J_{\lambda}^{*}(x ; 2)$ which can be obtained from [9, (18) and (117)]; the zonal polynomials $Z_{\lambda}$ in $[9,(18)$ and (116)] have the same normalization as the $J_{\lambda}$ so that $J_{\lambda}(x ; 2)=Z_{\lambda}(x)$. If $k=1$ the Jack polynomials reduce to the Schur functions $s_{\lambda}$, up to normalization. (For these remarks see [30, Proposition 1.2].)

As in Macdonald's manuscript [24] we write

$$
J_{\lambda}^{*}=J_{\lambda}^{*}\left(x ; k^{-1}\right)=J_{\lambda}\left(x ; k^{-1}\right) /\left\langle J_{\lambda}\left(x ; k^{-1}\right), J_{\lambda}\left(x ; k^{-1}\right)\right\rangle_{k}
$$

and

$$
\Omega_{\lambda}=\Omega_{\lambda}\left(x ; k^{-1}\right)=J_{\lambda}\left(x ; k^{-1}\right) / J_{\lambda}\left(1_{n} ; k^{-1}\right),
$$

where by $1_{n}$ we mean $x_{1}=x_{2}=\cdots=x_{n}=1$. Next we recall the Pochhammer symbol

$$
(a)_{s}=a(a+1) \cdots(a+s-1), \quad a \in \mathbf{C}, s \in \mathbf{Z}^{+},
$$

and define for a partition $\lambda=\left(\lambda_{1}, \lambda_{2}, \ldots, \lambda_{n}\right)$ of length $l(\lambda) \leq n$,

$$
(a)_{\lambda}=\prod_{i=1}^{n}(a-k(i-1))_{\lambda_{i}}, \quad a \in \mathbf{C} .
$$

Note that we suppress the dependence on $k$ of $(a)_{\lambda}$ in the notation.

We are now ready to define the generalized ${ }_{2} F_{1}$ hypergeometric function of matrix argument. For $a, b, c \in \mathbf{C}$ such that $(c)_{\lambda} \neq 0$ for all $\lambda$ we define

$$
{ }_{2} F_{1}\left(a, b ; c ; x ; k^{-1}\right)=\sum_{\lambda} \frac{(a)_{\lambda}(b)_{\lambda}}{(c)_{\lambda} k^{|\lambda|}} J_{\lambda}^{*}\left(x ; k^{-1}\right)
$$

where the sum is over all partitions $\lambda$ of length $\leq n$. This definition and its 
extension to ${ }_{p} F_{q}$ 's was given independently by Korányi $[15, \S \S 3-4]$ and Macdonald $[24,(6.4)]$. In $[15,(4.2)]$ Koranyi defines the $(a)_{\lambda}$ in terms of generalized gamma-functions which in turn are given as a certain extension of the Selberg integral. In doing so he follows the classical case (i.e. $k=\frac{1}{2}$ ) where a wellknown result on zonal polynomials (see e.g. [26, Theorem 7.2.10]) written in polar coordinates leads in a natural way to the extension of the Selberg integral for $k=\frac{1}{2}$. For general $k$ the extension has been studied by Kadell. Conjecture 2 in [11] states that there exist homogeneous symmetric polynomials such that if the integrand of Selberg's integral is multiplied by these polynomials then the integral has a given closed form. Macdonald observed that these symmetric polynomials should be the Jack polynomials $J_{\lambda}$, which led to conjecture C5 in [21]. As stated in Remark 3 the Jack polynomials indeed reduce to the zonal polynomials for $k=\frac{1}{2}$. Kadell established the conjecture in [12] while Macdonald proves a $q$-analogue in [20, Chapter IV]. The result leads to the coefficients $(a)_{\lambda}$ as defined in (2.9). Constantine $[2, \S 5]$ was the first to give definition (2.10) in the case $k=\frac{1}{2}$. In order to obtain [2, (25)] from (2.10) one needs the results stated in Remark 3 . James $[9, \S 8]$ also defines the ${ }_{p} F_{q}$ 's for the complex case, i.e. $k=1$. In [5, 5.3(1) and 6.1(1)] the real, complex and quaternionic case $(k=2)$ are treated simultaneously; they use the notation $Z_{\lambda}$ instead of $C_{\lambda}$ (sic!). In [4] hypergeometric functions ${ }_{p} F_{q}$ are associated with any so-called symmetric cone; as special cases they obtain the cone of real, complex and quaternionic positive definite matrices.

Now for $k=\frac{1}{2}$ Muirhead [25] (also see [26, §7.5]) proved that the ${ }_{2} F_{1}$ as defined in (2.10) satisfies a system of $n$ partial differential equations. Korányi $[15, \S 4]$ states that Muirhead's arguments can be generalized to show that the same result holds for the general parameter $k$. This statement is proven by Yan in [33, §2] (also see [32, Theorem A]); the proof follows closely that of the case $k=\frac{1}{2}$. To describe the result we define for $i=1,2, \ldots, n$ the operators $\Delta_{i}(a, b, c ; k)$ by

$$
\begin{aligned}
\Delta_{i}(a, b, c ; k)= & x_{i}\left(1-x_{i}\right) \partial_{x_{i}}^{2}+\left(c-k(n-1)-(a+b+1-k(n-1)) x_{i}\right) \partial_{x_{i}} \\
& +k \sum_{j=1 ; j \neq i}^{n} \frac{x_{i}\left(1-x_{i}\right)}{\left(x_{i}-x_{j}\right)} \partial_{x_{i}}-k \sum_{j=1 ; j \neq i}^{n} \frac{x_{j}\left(1-x_{j}\right)}{\left(x_{i}-x_{j}\right)} \partial_{x_{j}}
\end{aligned}
$$

where we have written $\partial_{x_{i}}=\partial / \partial x_{i}, \partial_{x_{i}}^{2}=\partial^{2} / \partial x_{i}^{2}$, etc. One now has the following result $[15, \S 4 ; 32$, Theorem $A ; 33, \S 2]$.

Theorem 2.2. The hypergeometric function ${ }_{2} F_{1}\left(a, b ; c ; x ; k^{-1}\right)$ is the unique symmetric function in the $n$ variables $x_{1}, \ldots, x_{n}$ that satisfies

$$
\Delta_{i}(a, b, c ; k) F=a b F, \quad i=1,2, \ldots, n,
$$

and which is analytic at $x_{1}=\cdots=x_{n}=0$ and normalized by $F(0)=1$.

Corollary 2.3. The hypergeometric function ${ }_{2} F_{1}\left(a, b ; c ; x ; k^{-1}\right)$ satisfies

$$
\Delta(a, b, c ; k){ }_{2} F_{1}=n a b_{2} F_{1}
$$


where $\Delta(a, b, c ; k)$ is given by

$$
\begin{aligned}
\Delta(a, b, c ; k)= & \sum_{i=1}^{n} \Delta_{i}(a, b, c ; k) \\
= & \sum_{i=1}^{n} x_{i}\left(1-x_{i}\right) \partial_{x_{i}}^{2}+2 k \sum_{i, j=1 ; i \neq j}^{n} \frac{x_{i}\left(1-x_{i}\right)}{\left(x_{i}-x_{j}\right)} \partial_{x_{i}} \\
& +\sum_{i=1}^{n}\left(c-k(n-1)-(a+b+1-k(n-1)) x_{i}\right) \partial_{x_{i}} .
\end{aligned}
$$

Macdonald [24] gives an independent proof of Corollary 2.3 along the same lines as the proof for $k=\frac{1}{2}$ by Muirhead [ 25 or 26, Theorem 7.5.4]. Note that the case $n=1$ reduces to the ordinary Gauss hypergeometric equation.

One can use Theorem 2.2 to prove that

$$
\begin{aligned}
& { }_{2} F_{1}\left(a, b ; c ; x_{1}, \ldots, x_{n} ; k^{-1}\right) \\
& \quad=\prod_{i=1}^{n}\left(1-x_{i}\right)^{-a}{ }_{2} F_{1}\left(c-b, a ; c ; \frac{x_{1}}{\left(x_{1}-1\right)}, \ldots, \frac{x_{n}}{\left(x_{n}-1\right)} ; k^{-1}\right) .
\end{aligned}
$$

In fact, if we put $u_{i}=x_{i} /\left(x_{i}-1\right)$ for $i=1, \ldots, n$ then it is not very hard to calculate the operators $\Delta_{i}$ in the $u$-coordinate and to show that the righthand side of (2.14) again satisfies the requirements of Theorem 2.2. This result is stated as Proposition 4.2 in [33] (also see [32, (3.3)]). The transformation properties of the operators $\Delta_{i}$ are treated systematically in [16].

Another result we will need later on is the generalized Gauss summation formula

$$
{ }_{2} F_{1}\left(a, b ; c ; 1_{n} ; k^{-1}\right)=\prod_{i=1}^{n} \frac{\Gamma(c-k(i-1)) \Gamma(c-a-b-k(i-1))}{\Gamma(c-a-k(i-1)) \Gamma(c-b-k(i-1))},
$$

which will certainly hold when $\operatorname{Re}(b-k(n-1))>0, \operatorname{Re}(c-b-k(n-1))>0$, $\operatorname{Re}(c-b-a-k(n-1))>0$ and $\operatorname{Re} k>-\min \left(\frac{1}{n}, \frac{\operatorname{Re}(b-k(n-1))}{n-1}, \frac{\operatorname{Re}(c-b-a-k(n-1))}{n-1}\right)$. Often the notation $\Gamma_{n}\left(c ; k^{-1}\right)=\gamma(k, n) \prod_{i=1}^{n} \Gamma(c-k(i-1))$ is introduced where $\gamma(k, n)$ is a constant which is author-dependent (see e.g. [24, $\S 6,32, \S 1$, $33, \S 4]$, and for the case $\left.k=\frac{1}{2}[26, \S 2.12]\right)$. The Gauss summation formula (2.15) is due to Macdonald and is obtained from the extension of the Selberg integral mentioned earlier. In fact, just as in the classical case (so $k=\frac{1}{2}$; see [26, Theorem 7.4.2]), Macdonald uses the extension to obtain an integral representation for the ${ }_{2} F_{1}\left(a, b ; c ; y_{1}, \ldots, y_{n} ; k^{-1}\right)$; this result is also stated by Yan [33, $\S 4(10)]$. Then Macdonald observes that one can take $y_{1}=\cdots=$ $y_{n}=1$ in this integral representation and then use the ordinary Selberg integral to obtain (2.15).

b. Root systems and Jacobi polynomial. Let $\left(e_{1}, e_{2}, \ldots, e_{n}\right)$ be the standard basis in $\mathbf{R}^{n}$ and let $\langle\cdot, \cdot\rangle$ denote the usual inner product for which this basis is orthonormal. Let $V$ denote the hyperplane in $\mathbf{R}^{n}$ orthogonal to the vector $e_{1}+e_{2}+\cdots+e_{n}$. The inner product on $\mathbf{R}^{n}$ induces an inner product on $V$ which we shall also denote by $\langle\cdot, \cdot\rangle$. We identify the dual space of $\mathbf{R}^{n}$ with $\mathbf{R}^{n}$ and the dual space of $V$ with $V$ by means of these inner products. We consider $\mathbf{R}^{n}$ as the standard real form of $\mathbf{C}^{n}$ and extend $\langle\cdot, \cdot\rangle$ to a complex bilinear form on 
$\mathrm{C}^{n}$. The complexification of $V$ in $\mathbf{C}^{n}$ will be denoted by $V_{\mathbf{C}}$. Again we identify their dual spaces by means of $\langle\cdot, \cdot\rangle$. We use $t_{1}, t_{2}, \ldots, t_{n}$ as coordinates with respect to $\left(e_{1}, \ldots, e_{n}\right)$. Then $V=\left\{\left(t_{1}, \ldots, t_{n}\right) \in \mathbf{R}^{n} \mid \sum_{i=1}^{n} t_{i}=0\right\}$.

In $V$ we consider the set of vectors

$$
R_{A}=\left\{ \pm\left(e_{i}-e_{j}\right) \mid 1 \leq i<j \leq n\right\} .
$$

This set forms a root system of type $A_{n-1}$ in $V$. We choose

$$
S_{A}=\left\{e_{1}-e_{2}, e_{2}-e_{3}, \ldots, e_{n-1}-e_{n}\right\}
$$

as basis for $R_{A}$ and we let $R_{A}^{+}$denote the set of positive roots relative to this choice. In $\mathbf{R}^{n}$ we consider the set of vectors

$$
R_{B}=\left\{ \pm e_{i}, \pm 2 e_{i}, \pm\left(e_{k} \pm e_{l}\right) \mid i=1, \ldots, n ; 1 \leq k<l \leq n\right\}
$$

which forms a root system of type $B C_{n}$. We choose

$$
S_{B}=\left\{e_{1}-e_{2}, e_{2}-e_{3}, \ldots, e_{n-1}-e_{n}, e_{n}\right\}
$$

as basis for $R_{B}$ and let $R_{B}^{+}$denote the corresponding set of positive roots. The Weyl group of $R_{X}$ will be denoted by $W_{X}$. Here we introduced the convention that $X$ stands for either $A$ or $B$. Let $P_{X}$ be the weight lattice of $R_{X}$. So

$$
P_{A}=\left\{\lambda \in V \mid 2\langle\lambda, \alpha\rangle /\langle\alpha, \alpha\rangle \in \mathbf{Z} \forall \alpha \in R_{A}\right\},
$$

and similarly for $P_{B}$. The set of dominant weights will be denoted by $P_{X}^{+}$:

$$
P_{X}^{+}=\left\{\lambda \in P_{X} \mid\langle\lambda, \alpha\rangle \geq 0 \forall \alpha \in R_{X}^{+}\right\} .
$$

If we write

$$
R_{B}^{0}=\left\{\alpha \in R_{B} \mid 2 \alpha \notin R_{B}\right\},
$$

then $R_{B}^{0}$ is a root system of type $C_{n}$. Denote by $\omega_{1}, \omega_{2}, \ldots, \omega_{n}$ the fundamental weights of $C_{n}$, so that for $i=1,2, \ldots, n$ one has

$$
\omega_{i}=e_{1}+e_{2}+\cdots+e_{i} \text {. }
$$

Then

$$
P_{B}=\mathbf{Z} \omega_{1}+\mathbf{Z} \omega_{2}+\cdots+\mathbf{Z} \omega_{n}=\mathbf{Z} e_{1}+\cdots+\mathbf{Z} e_{n}=\mathbf{Z}^{n}
$$

and

$$
P_{B}^{+}=\mathbf{Z}^{+} \omega_{1}+\mathbf{Z}^{+} \omega_{2}+\cdots+\mathbf{Z}^{+} \omega_{n}
$$

Note that

$$
R_{A}=\left\{\alpha \in R_{B} \mid\left\langle\alpha, \omega_{n}\right\rangle=0\right\}, \quad S_{A}=\left\{\alpha \in S_{B} \mid\left\langle\alpha, \omega_{n}\right\rangle=0\right\},
$$

and that we can consider $W_{A}$ as subgroup of $W_{B}$. We let $\pi$ denote the orthogonal projection along $\omega_{n}$ onto $V$, then

$$
\pi(t)=t-\frac{1}{n}\left(\sum_{i=1}^{n} t_{i}\right) \omega_{n}, \quad t=\left(t_{1}, \ldots, t_{n}\right) \in \mathbf{R}^{n} .
$$

Since $S_{A}=\left\{\alpha \in S_{B} \mid \pi(\alpha)=\alpha\right\}$ it follows that $\left\{\pi\left(\omega_{i}\right) \mid i=1,2, \ldots, n-1\right\}$ is the set of fundamental weights for $A_{n-1}$ so that

$$
P_{A}=\pi\left(P_{B}\right) .
$$


Also $\pi \circ w=w \circ \pi$ for all $w \in W_{A}$. Let $Q_{X}$ be the root lattice $\mathbf{Z} R_{X}$ and put $Q_{X}^{+}=\mathbf{Z}^{+} R_{X}^{+}$. Then

$$
Q_{B}=P_{B}=\mathbf{Z}^{n}, \quad Q_{A}=V \cap \mathbf{Z}^{n} \subset P_{A}
$$

and also

$$
Q_{A}^{+}=V \cap Q_{B}^{+}
$$

(if $\lambda=\sum_{i=1}^{n-1} b_{i}\left(e_{i}-e_{i+1}\right)+b_{n} e_{n} \in Q_{B}^{+}$and $\lambda \in V$ then $b_{n}=0$ ).

Next we want to introduce the tori corresponding to the $Q$-lattices. In general, a compact torus $T_{L}$ is a Lie group of the form $\mathbf{R}^{n} / L$ where $L \subset \mathbf{R}^{n}$ is a lattice of rank $n$. Choose a Z-basis $\varepsilon_{1}, \ldots, \varepsilon_{n}$ of $L$, then $D=\left\{\sum_{i=1}^{n} t_{i} \varepsilon_{i} \mid 0<\right.$ $\left.t_{i}<1\right\}$ is an open dense subset of a fundamental domain. Normalized Haar measure on $T_{L}$ is then given by

$$
\int_{T_{L}} f(t) d t=\int_{D} f(v) \frac{d v}{\operatorname{vol} L},
$$

where $d v$ is Lebesgue measure and $\operatorname{vol} L=\operatorname{vol} D$ is independent of the choice of a basis. By looking at it this way it follows easily that for a surjective continuous group homomorphism $\phi: T_{L_{1}} \rightarrow T_{L_{2}}$ one has

$$
\int_{T_{L_{2}}} f(t) d t=\int_{T_{L_{1}}}(f \circ \phi)\left(t^{\prime}\right) d t^{\prime}, \quad f \in C\left(T_{L_{2}}\right) .
$$

Now let $T_{A}$ and $T_{B}$ be the compact tori defined by

$$
T_{B}=i \mathbf{R}^{n} / 2 \pi i Q_{B}=i \mathbf{R}^{n} / 2 \pi i \mathbf{Z}^{n}
$$

and

$$
T_{A}=i V / 2 \pi i Q_{A}=i V / 2 \pi i\left(V \cap \mathbf{Z}^{n}\right) .
$$

Later on we will also consider their complexifications $H_{A}$ and $H_{B}$, i.e.

$$
H_{B}=\mathbf{C}^{n} / 2 \pi i Q_{B}=\mathbf{C}^{n} / 2 \pi i \mathbf{Z}^{n}
$$

and

$$
H_{A}=V_{\mathbf{C}} / 2 \pi i Q_{A}=V_{\mathbf{C}} / 2 \pi i\left(V_{\mathbf{C}} \cap \mathbf{Z}^{n}\right) .
$$

We use the map

$$
\sum_{j=1}^{n} t_{j} e_{j} \quad\left(\bmod 2 \pi i \mathbf{Z}^{n}\right) \rightarrow\left(e^{t_{1}}, \ldots, e^{t_{n}}\right) \quad\left(t_{j} \in i \mathbf{R} \forall j\right)
$$

to identify $T_{B}$ with $\left\{\left(x_{1}, \ldots, x_{n}\right) \in \mathbf{C}^{n}|| x_{j} \mid=1 \forall j\right\}$. It follows that $T_{A}=\{x \in$ $\left.T_{B} \mid x_{1} x_{2} \cdots x_{n}=1\right\}$. Consider the group homomorphism defined by

$$
\begin{gathered}
\phi: T_{A} \times i \mathbf{R} / 2 \pi i \mathbf{Z} \rightarrow T_{B}, \\
\left(\left(t_{1}, \ldots, t_{n}\right)^{\circ}, \dot{t}\right) \rightarrow\left(t_{1}+t, t_{2}+t, \ldots, t_{n}+t\right)^{\circ},
\end{gathered}
$$

where ()$^{\bullet}$ denotes equivalence class; multiplicatively $\phi$ is given by

$$
\left(\left(x_{1}, \ldots, x_{n}\right), x\right) \in T_{A} \times S^{1} \rightarrow\left(x x_{1}, x x_{2}, \ldots, x x_{n}\right) \in T_{B} .
$$

From (2.19) then follows that for $f \in C\left(T_{B}\right)$ we have

$$
\int_{T_{B}} f(x) d x=\int_{s \in S^{1}} \int_{x^{\prime} \in T_{A}} f\left(s x_{1}^{\prime}, s x_{2}^{\prime}, \ldots, s x_{n}^{\prime}\right) d x^{\prime} d s,
$$


where $d x^{\prime}, d s$ and $d x$ are normalized Haar measure on $T_{A}, S^{1}$ and $T_{B}$ respectively.

Let $\mathbf{C}\left[P_{B}\right]$ denote the group algebra over $\mathbf{C}$ of $P_{B}$. If $\lambda \in P_{B}$ then we write $e^{\lambda}$ for the corresponding element of $\mathrm{C}\left[P_{B}\right]$. Multiplication in $\mathrm{C}\left[P_{B}\right]$ is defined as $e^{\lambda} e^{\mu}=e^{\lambda+\mu}$ for $\lambda, \mu \in P_{B}$. This group algebra $\mathbf{C}\left[P_{B}\right]$ can also be considered (and we will do so) as the algebra of functions on $H_{B}$ generated over $\mathbf{C}$ by the exponentials $e^{\lambda}$ with $\lambda \in P_{B}$. Here $e^{\lambda}$ denotes the function $e^{\lambda}(\dot{t})=e^{\langle\lambda, t\rangle}$ where $t \in \mathbf{C}^{n}$ (when restricted to $T_{B}, e^{\lambda}$ is the character corresponding to $\lambda$ ). In particular $e^{e_{i}}(\dot{t})=e^{t_{i}}$ if $t=\left(t_{1}, \ldots, t_{n}\right) \in \mathbf{C}^{n}$. An element $f \in \mathbf{C}\left[P_{B}\right]$ will be called exponential polynomial; it is a finite sum of the form

$$
f=\sum_{\lambda \in P_{B}} c_{\lambda} e^{\lambda}
$$

The support of $f$ is the (finite) set $\left\{\lambda \in P_{B} \mid c_{\lambda} \neq 0\right\}$. The Weyl group $W_{B}$ acts on $P_{B}$ and hence also on $\mathrm{C}\left[P_{B}\right]$. An element $x \in \mathbf{C}\left[P_{B}\right]$ is called invariant (under $W_{B}$ ) if $w \cdot f=f$ for all $w \in W_{B}$ and it is called skew-invariant if $w \cdot f=\operatorname{det} w \cdot f$ for all $w \in W_{B}$. We write $\mathbf{C}\left[P_{B}\right]^{W_{B}}$ for the subalgebra of $\mathrm{C}\left[P_{B}\right]$ consisting of all $W_{B}$-invariant elements in $\mathbf{C}\left[P_{B}\right]$. An invariant element $f \in \mathbf{C}\left[P_{B}\right]$ can be written as

$$
f=\sum_{\lambda \in P_{B}^{+}} c_{\lambda} m_{\lambda}^{B}
$$

where $m_{\lambda}^{B}$ is defined as

$$
m_{\lambda}^{B}=\sum_{\mu \in W_{B} \cdot \lambda} e^{\mu}, \quad \lambda \in P_{B}^{+}
$$

Here $W_{B} \cdot \lambda$ denotes the $W_{B}$-orbit of $\lambda$. The analogous concepts for $\left(P_{A}, W_{A}\right)$ will be denoted by $\mathbf{C}\left[P_{A}\right], \mathbf{C}\left[P_{A}\right]^{W_{A}}$ and $m_{\lambda}^{A}$; these will be considered as functions on $H_{A}$.

We now introduce the Jacobi polynomials associated with the root system $R_{X}$ (as always $X$ stands for either $A$ or $B$ ). Our reference for this material is $[6, \S \S 2-3,7, \S 8]$. For $\lambda \in P_{X}^{+}$we write $\Pi_{X}(\lambda)$ for the convex hull of the orbit $W_{X} \cdot \lambda$ intersected with $\lambda+Q_{X}$. Note that $\Pi_{X}(\lambda)$ is equal to the set of all $\mu \in P_{X}^{+}$with $\lambda-\mu \in Q_{X}^{+}$and the $W_{X}$-conjugates of such $\mu$. Let $m$ be the number of $W_{X}$-orbits in $R_{X}$. Define $\mathscr{K} \cong \mathbf{C}^{m}$ as the vector space of $W_{X}$-invariant functions on $R_{X}$ with values in $\mathbf{C}$. We call elements of $\mathscr{K}$ multiplicity functions on $R_{X}$ and we denote by $k_{\alpha}$ the value of $\kappa \in \mathscr{K}$ on $\alpha \in R_{X}$. For the $A_{n-1}$-case there is only one $W_{A}$-orbit so that we will simply write $k=k_{\alpha}$ for all $\alpha \in R_{A}$. For the $B C_{n}$-case there are three $W_{B}$-orbits; we will write

$$
k_{1}=k_{e_{i}}, \quad k_{2}=k_{2 e_{i}}, \quad k_{3}=k_{e_{i} \pm e_{j}} .
$$

Introduce the following (multivalued) function on $H_{X}$ :

$$
\delta_{X}(\kappa)=\prod_{\alpha \in R_{X}^{+}}\left(e^{\frac{1}{2} \alpha}-e^{-\frac{1}{2} \alpha}\right)^{2 k_{\alpha}}, \quad \kappa \in \mathscr{K} .
$$

Now let $\kappa \in \mathscr{K}$ be such that $k_{\alpha} \geq 0$ for all $\alpha \in R_{X}$. We endow $\mathbf{C}\left[P_{X}\right]^{W_{X}}$ with a (Hermitian) inner product as follows:

$$
\langle f, g\rangle_{X, \kappa}=\int_{T_{X}} f(x) \overline{g(x)}\left|\delta_{X}(\kappa)(x)\right| d x, \quad f, g \in \mathbf{C}\left[P_{X}\right]^{W_{X}}
$$


Note that $\prod_{i<j}\left(e^{\frac{1}{2}\left(e_{i}-e_{j}\right)}-e^{-\frac{1}{2}\left(e_{i}-e_{j}\right)}\right)=\prod_{i<j}\left(e^{e_{i}}-e^{e_{j}}\right)$ as functions on $T_{A}$ so that

$$
\langle f, g\rangle_{A, \kappa}=\int_{T_{A}} f(x) \overline{g(x)} \prod_{i<j}\left|x_{i}-x_{j}\right|^{2 k} d x, \quad k \geq 0 .
$$

Define the Jacobi polynomials $P_{X}(\lambda, \kappa)$ on $H_{X}\left(\lambda \in P_{X}^{+}\right)$associated with $R_{X}$ and multiplicity function $\kappa \in \mathscr{K}$ by means of the following two properties:

1. $P_{X}(\lambda, \kappa)=\sum_{\mu \in \Pi_{X}(\lambda)} \Gamma_{\mu}(\lambda, \kappa) e^{\mu}$ with $\Gamma_{\lambda}(\lambda, \kappa)=1$ and $\Gamma_{w \mu}(\lambda, \kappa)=$ $\Gamma_{\mu}(\lambda, \kappa)$ for all $w \in W_{X}$.

2. $\left\langle P_{X}(\mu, \kappa), P_{X}(\nu, \kappa)\right\rangle_{X, \kappa}=0$ for all $\nu \in P_{X}^{+}$with $\mu-\nu \in Q_{X}^{+}$.

Note that in [6, Definition 3.13; 7, $\S 8$ and 28, §2] the Jacobi polynomials are parametrized by $\mu=w_{0} \lambda \in P_{X}^{-}$instead of $\lambda \in P_{X}^{+}$. Here $w_{0}$ denotes the longest Weyl group element. For the case $X=B$ we have $w_{0}=-1$.

One may replace 2 by the condition that $P_{X}(\lambda, \kappa)$ satisfies

$$
L_{X}(\kappa) P_{X}(\lambda, \kappa)=\left\langle\lambda, \lambda+2 \rho_{X}(\kappa)\right\rangle P_{X}(\lambda, \kappa),
$$

where

$$
\rho_{X}(\kappa)=\frac{1}{2} \sum_{\alpha \in R_{X}^{+}} k_{\alpha} \alpha
$$

and $L_{X}(\kappa)$ is the so-called generalized radial part of the Laplace-Beltrami operator. It is given by

$$
L_{X}(\kappa)=L_{X}(0)+\sum_{\alpha \in R_{X}^{+}} k_{\alpha} \operatorname{coth} \frac{1}{2} \alpha \cdot \partial_{\alpha},
$$

where $L_{X}(0)$ denotes the ordinary Laplacian on $\mathbf{R}^{n}$ (in the case $X=B$ ) or on $V$ (in the case $X=A$ ) and $\partial_{\alpha}$ denotes differentiation in the direction of $\alpha$. We also note that because of the Weyl group invariance of $P_{X}(\lambda, \kappa)$ one may write

$$
P_{X}(\lambda, \kappa)=\sum_{\mu \in P_{X}^{+}, \lambda-\mu \in Q_{X}^{+}} d_{\lambda \mu} m_{\mu}^{X}
$$

with $m_{\mu}^{X}$ as defined in (2.21) and $d_{\lambda \lambda}=1$. The condition $\lambda-\mu \in Q_{X}^{+}$is often denoted by $\mu \prec \lambda$, which gives the usual partial ordering on weights. In order to avoid confusion with $\leq$ for partitions we do not use this notation.

Finally we define for $\bar{\lambda} \in \mathbf{C}^{n}$ and $\kappa \in \mathscr{K}$ the generalized Harish-Chandra c-function by

$$
\mathbf{c}_{X}(\lambda, \kappa)=\prod_{\alpha \in R_{X}^{+}} \frac{\Gamma\left(\left\langle\lambda, \alpha^{\vee}\right\rangle+\frac{1}{2} k_{\frac{\alpha}{2}}\right) \Gamma\left(\left\langle\rho_{X}(\kappa), \alpha^{\vee}\right\rangle+\frac{1}{2} k_{\frac{\alpha}{2}}+k_{\alpha}\right)}{\Gamma\left(\left\langle\lambda, \alpha^{\vee}\right\rangle+\frac{1}{2} k_{\frac{\alpha}{2}}+k_{\alpha}\right) \Gamma\left(\left\langle\rho_{X}(\kappa), \alpha^{\vee}\right\rangle+\frac{1}{2} k_{\frac{\alpha}{2}}\right)}
$$

where $\Gamma$ denotes the usual gamma-function and $\alpha^{\vee}=2 \alpha /\langle\alpha, \alpha\rangle$ (we should warn the reader that this definition differs from $[6, \S 6$ and $28, \S 2]$ by a change of $\lambda$ into $-\lambda$ ). For specific values of the $k_{\alpha}$ this is the well-known product formula of Gindikin and Karpelevich for the classical Harish-Chandra c-function. Later it will be convenient to have $\rho_{B}(\kappa)$ explicit; with the convention (2.22) one has

$$
2 \rho_{B}(\kappa)=\left(k_{1}+2 k_{2}\right)\left(\sum_{i=1}^{n} e_{i}\right)+2 k_{3} \sum_{i=1}^{n}(n-i) e_{i}
$$




\section{The CONNECTION BeTWEen JaCK AND JaCOBI POLYNOMIALS}

In this section we want to describe the precise relationship between Jack and Jacobi polynomials. In order to do so we first study in some detail the relationship between partitions and weights.

We associate with a partition $\lambda=\left(\lambda_{1}, \lambda_{2}, \ldots, \lambda_{n}\right)$ of length $l(\lambda) \leq n$ the vector $\lambda=\lambda_{1} e_{1}+\lambda_{2} e_{2}+\cdots+\lambda_{n} e_{n}$ in $\mathbf{R}^{n}$. By (2.16) we have $\lambda=\left(\lambda_{1}-\lambda_{2}\right) \omega_{1}+$ $\left(\lambda_{2}-\lambda_{3}\right) \omega_{2}+\cdots+\left(\lambda_{n-1}-\lambda_{n}\right) \omega_{n-1}+\lambda_{n} \omega_{n}$ so that $\lambda \in P_{B}^{+}$. On the other hand, if $\lambda \in P_{B}^{+}$then $\lambda=a_{1} \omega_{1}+\cdots+a_{n} \omega_{n}$ with $a_{i} \in \mathbf{Z}^{+}$so that by (2.16) we have $\lambda=\lambda_{1} e_{1}+\cdots+\lambda_{n} e_{n}$ with $\lambda_{i}=a_{1}+\cdots+a_{i}$, which determines a unique partition $\left(\lambda_{1}, \ldots, \lambda_{n}\right)$. From the partition $\lambda=\left(\lambda_{1}, \ldots, \lambda_{n}\right)$ we also obtain

$$
\pi(\lambda)=\left(\lambda_{1}-\lambda_{2}\right) \pi\left(\omega_{1}\right)+\cdots+\left(\lambda_{n-1}-\lambda_{n}\right) \pi\left(\omega_{n-1}\right) \in P_{A}^{+}
$$

where, as before, $\pi$ is the orthogonal projection along $\omega_{n}$. The partitions $\lambda=\left(\lambda_{1}, \ldots, \lambda_{n}\right)$ and $\lambda+a(1, \ldots, 1)=\left(\lambda_{1}+a, \ldots, \lambda_{n}+a\right)\left(a \in \mathbf{Z}^{+}\right.$and $a \geq-\lambda_{n}$ ) correspond of course to the same weight in $P_{A}^{+}$. One obtains a one-to-one correspondence between weights in $P_{A}^{+}$and partitions if we fix the weight of the partitions. Let us extend the notion of the weight of a partition to any vector in $\mathbf{R}^{n}$; so for $\lambda=\lambda_{1} e_{1}+\cdots+\lambda_{n} e_{n}$ in $\mathbf{R}^{n}$ we put $|\lambda|=\sum_{i=1}^{n} \lambda_{i}$. We then write

$$
P_{d}=\left\{\lambda=\lambda_{1} e_{1}+\cdots+\lambda_{n} e_{n} \in P_{B}|| \lambda \mid=d\right\}, \quad d \in \mathbf{Z} .
$$

For $\lambda, \mu \in P_{d}$ one has of course $\pi(\lambda)-\pi(\mu)=\lambda-\mu$ so that $\pi$ is one-to-one on $P_{d}$. Now let $\lambda$ and $\mu$ be partitions with $|\lambda|=|\mu|$. This gives us $\lambda, \mu \in P_{B}^{+}$ with $|\lambda|=|\mu|$ and hence

$$
\lambda-\mu=\sum_{i=1}^{n-1}\left(\sum_{j=1}^{i}\left(\lambda_{j}-\mu_{j}\right)\right)\left(e_{i}-e_{i+1}\right) \in Q_{A} \subset Q_{B} .
$$

Now assume that $\mu \leq \lambda$ as partitions so that $\sum_{j=1}^{i} \mu_{j} \leq \sum_{j=1}^{i} \lambda_{j}$ for all $i \geq 1$ and equality holds for $i=n$. Then we see from (3.1) that $\lambda-\mu \in Q_{A}^{+} \subset Q_{B}^{+}$. If on the other hand $\lambda-\mu \in Q_{B}^{+}$with $\lambda, \mu \in P_{B}^{+}$and $|\lambda|=|\mu|$ then by (2.18) we have $\lambda-\mu \in Q_{A}^{+}$and since $\lambda_{j}-\mu_{j} \geq 0$ for all $j$ it follows from (3.1) that $\mu \leq \lambda$ as partitions. Finally we note that if $\mu \subset \lambda$ as partitions then $\lambda-\mu=\sum_{i=1}^{n}\left(\lambda_{i}-\mu_{i}\right) e_{i} \in Q_{B}^{+}$. Hence we have obtained:

Lemma 3.1. (i) Let $\mu$ and $\lambda$ be partitions with $|\lambda|=|\mu|$. Then $\pi(\lambda)-\pi(\mu)=$ $\lambda-\mu$ and

$$
\mu \leq \lambda \text { as partitions } \Leftrightarrow \lambda-\mu \in Q_{A}^{+} \Leftrightarrow \lambda-\mu \in Q_{B}^{+} \text {. }
$$

(ii) If $\mu$ and $\lambda$ are partitions such that $\mu \subset \lambda$ then $\lambda-\mu \in Q_{B}^{+}$.

For future reference we prove here the following result.

Proposition 3.2. Let $\lambda$ be a partition which we also consider as element of $P_{B}^{+}$. Then

\{partitions $\nu \mid \exists$ partition $\mu$ with $\nu \leq \mu, \mu \subset \lambda\}=\left\{\nu \in P_{B}^{+} \mid \lambda-\nu \in Q_{B}^{+}\right\}$.

Remark. The set $\left\{\nu \in P_{B}^{+} \mid \lambda-\nu \in Q_{B}^{+}\right\}$is precisely the intersection of $\Pi_{B}(\lambda)$ with the positive Weyl chamber of $B C_{n}$.

Proof. The inclusion $\subset$ is obvious since $\mu \subset \lambda$ implies that $\lambda-\mu \in Q_{B}^{+}$while $\nu \leq \mu$ as partitions also implies $\mu-\nu \in Q_{B}^{+}$(Lemma 3.1). Hence $\lambda-\nu \in Q_{B}^{+}$. 
Next we prove the other inclusion. So let $\nu=\sum_{i=1}^{n} \nu_{i} e_{i}$ in $P_{B}^{+}$with $\lambda-\nu \in Q_{B}^{+}$. Write $\lambda=\sum_{i=1}^{n} \lambda_{i} e_{i}$. We have to show that $\exists \mu=\sum_{i=1}^{n} \mu_{i} e_{i}$ in $P_{B}^{+}$with $\mu \subset \lambda$, i.e. $\mu_{i} \leq \lambda_{i}$ and such that $\mu-\nu \in Q_{A}^{+}$(since then $|\mu|=|\nu|$ and hence also $\nu \leq \mu$ as partitions by Lemma 3.1). We know that

$$
\lambda=\nu+\sum_{i=1}^{n-1} a_{i}\left(e_{i}-e_{i+1}\right)+a_{n} e_{n}, \quad a_{i} \in \mathbf{Z}^{+} .
$$

Put $a_{0}=0$ and let $i \in\{1,2, \ldots, n\}$. Since $\lambda_{i} \geq 0$ one has $\nu_{i}+a_{i}-a_{i-1} \geq 0$ so that from $\nu_{i}+\cdots+\nu_{n}<a_{i-1}$ it would follow that $\nu_{i+1}+\nu_{i+2}+\cdots+\nu_{n}<$ $a_{i-1}-\nu_{i} \leq a_{i}$ and similarly $\nu_{i+2}+\cdots+\nu_{n}<a_{i+1}, \ldots, \nu_{n}<a_{n-1}$. So, starting with $\nu_{1}+\cdots+\nu_{n} \geq 0=a_{0}$, we can choose $i \in\{1,2, \ldots, n\}$ such that

$$
\begin{aligned}
& \nu_{i}+\nu_{i+1}+\cdots+\nu_{n} \geq a_{i-1} \text { and } \\
& \nu_{j}+\nu_{j+1}+\cdots+\nu_{n}<a_{j-1} \text { for } j=i+1, \ldots, n \text {. }
\end{aligned}
$$

Now define

$$
\begin{aligned}
\mu= & \nu+a_{1}\left(e_{1}-e_{2}\right)+\cdots+a_{i-1}\left(e_{i-1}-e_{i}\right) \\
& +\left(\nu_{i+1}+\cdots+\nu_{n}\right)\left(e_{i}-e_{i+1}\right)+\cdots+\nu_{n}\left(e_{n-1}-e_{n}\right)
\end{aligned}
$$

then

$$
\mu_{j}= \begin{cases}\lambda_{j}, & j=1,2, \ldots, i-1 ; \\ \nu_{i}+\nu_{i+1}+\cdots+\nu_{n}-a_{i-1}, & j=i \\ 0, & j=i+1, \ldots, n .\end{cases}
$$

Since by $(3.2)$

$$
\lambda_{i-1} \geq \lambda_{i}=\nu_{i}+a_{i}-a_{i-1}>\nu_{i}+\nu_{i+1}+\cdots+\nu_{n}-a_{i-1} \geq 0
$$

we see that $\mu$ is indeed a partition, i.e. $\mu \in P_{B}^{+}$. Furthermore, since $a_{i} \in Z^{+}$ and $\nu_{i} \in \mathbf{Z}^{+}$for all $i$ it is clear that $\mu-\nu \in Q_{A}^{+}$. It remains to show that $\mu \subset \lambda$, i.e. $\lambda_{j}-\mu_{j} \geq 0$ for all $j$. This is clear for $j \neq i$ while for $j=i$ we have

$$
\lambda_{i}-\mu_{i}=\left(\nu_{i}+a_{i}-a_{i-1}\right)-\left(\nu_{i}+\cdots+\nu_{n}-a_{i-1}\right)=a_{i}-\left(\nu_{i+1}+\cdots+\nu_{n}\right)
$$

which is nonnegative by (3.2) again. This proves the proposition.

As in $\S 2 \mathrm{~b}$ we now take $e^{\lambda} \in \mathbf{C}\left[P_{B}\right]$ and consider it as function on $T_{B}$. By restriction we can view it as function on $T_{A}$ and we will denote this restriction by $\left(e^{\lambda}\right)^{\sim}$. One has

$$
\left(e^{\lambda}\right)^{\sim}=e^{\pi(\lambda)}
$$

since $\pi$ is the orthogonal projection onto $V$ and $\pi\left(\mathbf{Z}^{n}\right)=V \cap \mathbf{Z}^{n}=Q_{A}$. We extend the restriction to $\mathbf{C}\left[P_{B}\right]$ by linearity so that from $f \in \mathbf{C}\left[P_{B}\right]$ we obtain $\tilde{f} \in \mathbf{C}\left[P_{A}\right]$. For $d \in \mathbf{Z}$ we write $\mathbf{C}\left[P_{B}\right]_{d}$ for the collection of $f=\sum_{\lambda} c_{\lambda} e^{\lambda}$ in $\mathbf{C}\left[P_{B}\right]$ in which the sum is taken over $\lambda \in P_{d}$. Then $f \rightarrow \tilde{f}$ is obviously injective on $\mathbf{C}\left[P_{B}\right]_{d}$. Note that since $\pi$ commutes with $W_{A}$ it follows that if $C$ is a $W_{A}$-invariant subset of $P_{B}$ then $\pi(C)$ is a $W_{A}$-invariant subset of $P_{A}$ (here $W_{A}$ is considered as subgroup of $W_{B}$ ). Hence if $f \in \mathbf{C}\left[P_{B}\right]$ is $W_{A^{-}}$ invariant then $\tilde{f} \in \mathbf{C}\left[P_{A}\right]^{W_{A}}$. Let $\lambda$ be any partition. The Jack polynomial 
$J_{\lambda}\left(x_{1}, \ldots, x_{n} ; k^{-1}\right)$ is a symmetric polynomial in $x_{1}, \ldots, x_{n}$ and homogeneous of degree $|\lambda|$. We put $x_{i}=e^{t_{i}}=e^{e_{i}}(\dot{t})$ for $t=\left(t_{1}, \ldots, t_{n}\right) \in \mathbf{C}^{n}$ and consider $J_{\lambda}\left(\lambda \in P_{B}^{+}\right)$as $W_{A}$-invariant element of $\mathbf{C}\left[P_{B}\right]_{|\lambda|}$ (cf. subsection $2 \mathrm{~b}$ ). The same applies to the monomial symmetric polynomial $m_{\lambda}=m_{\lambda}\left(x_{1}, \ldots, x_{n}\right)$. From the definitions of $m_{\lambda}$ (in (2.3)) and $m_{\mu}^{A}$ (cf. (2.21)) and the fact that $\pi\left(W_{A} \cdot \lambda\right)=W_{A} \cdot \pi(\lambda)$ it follows immediately that

$$
\tilde{m}_{\lambda}=m_{\pi(\lambda)}^{A} \text {. }
$$

On $\mathbf{C}\left[P_{B}\right]_{d}$ we introduce an inner product $\langle\cdot, \cdot\rangle_{A_{e}, k}$-which is an 'extended version' of the inner product $\langle\cdot, \cdot\rangle_{A, k}$ in $(2.25)$-by

$$
\langle f, g\rangle_{A_{e}, k}=\int_{T_{B}} f(x) \overline{g(x)} \prod_{i<j}\left|x_{i}-x_{j}\right|^{2 k} d x, \quad k \geq 0 .
$$

For any partition $\lambda$ of length $l(\lambda) \leq n$ we define $h_{*}(\lambda)$ by

$$
h_{*}(\lambda)=\prod_{(i, j) \in \lambda}\left(\lambda_{j}^{\prime}-i+1+k^{-1}\left(\lambda_{i}-j\right)\right)
$$

and $h^{*}(\lambda)$ by

$$
h^{*}(\lambda)=\prod_{(i, j) \in \lambda}\left(\lambda_{j}^{\prime}-i+k^{-1}\left(\lambda_{i}-j+1\right)\right) .
$$

The factors in (3.6) are the so-called 'lower hook-lengths' at $(i, j) \in \lambda$ while the factors in (3.7) are the so-called 'upper hook-lengths' at $(i, j) \in \lambda$ (cf. $[30, \S 5$ or $21, \S 4])$; they both reduce to the ordinary hook-length at $(i, j)$ if $k=1$. We can now state the exact relationship between the Jack and the Jacobi polynomials.

Proposition 3.3. Consider the Jack polynomial $J_{\lambda}\left(x ; k^{-1}\right)$ as function on $T_{B}$ under the change of coordinates $x_{i}=e^{t_{i}}=e^{e_{i}}(\dot{t})$ if $t=\left(t_{1}, \ldots, t_{n}\right) \in \mathbf{C}^{n}$. Let $P_{A}(\mu, k)$ denote the Jacobi polynomial associated with $A_{n-1}$. Then

$$
\widetilde{J}_{\lambda}\left(\cdot ; k^{-1}\right)=h_{*}(\lambda) P_{A}(\pi(\lambda), k) .
$$

Proof. Let $x^{\prime}=\left(x_{1}, \ldots, x_{n}\right) \in T_{A}$ and $s \in S^{1}$ (see subsection 2b). Then $f\left(s x^{\prime}\right)=s^{d} f\left(x^{\prime}\right)$ for $f \in \mathbf{C}\left[P_{B}\right]_{d}$ and $\left|\prod_{i<j}\left(s x_{i}-s x_{j}\right)\right|=\left|\prod_{i<j}\left(x_{i}-x_{j}\right)\right|$. Hence

$$
\langle f, g\rangle_{A_{e}, k}=\langle\tilde{f}, \tilde{g}\rangle_{A, k}, \quad f, g \in \mathbf{C}\left[P_{B}\right]_{d} .
$$

In [21] (also see [20, Chapter VI, §§9-10]) Macdonald notes that the Jack polynomials $J_{\lambda}$ are also characterized (up to normalization) as the symmetric polynomials of the form (2.6) that satisfy

$$
\left\langle J_{\lambda}, J_{\mu}\right\rangle_{A_{e}, k}=0, \quad \mu<\lambda .
$$

By restriction to $T_{A}$ we obtain $W_{A}$-invariant polynomials $\widetilde{J}_{\lambda}$ in $\mathbf{C}\left[P_{B}\right]_{|\lambda|}$ which by (3.9) will satisfy

$$
\left\langle\widetilde{J}_{\lambda}, \widetilde{J}_{\mu}\right\rangle_{A, k}=0, \quad \mu<\lambda .
$$

Moreover, by (2.6), (3.4) and Lemma 3.1 they will have the form

$$
\widetilde{J}_{\lambda} \sum_{\nu \in P_{A}^{+}, \nu-\pi(\lambda) \in Q_{A}^{+}} c_{\lambda \nu} m_{\nu}^{A}
$$


But these two properties characterize exactly the Jacobi polynomials $P_{A}(\pi(\lambda), k)$, up to normalization (cf. subsection 2b). Now the coefficient of $m_{\pi(\lambda)}^{A}$ in $P_{A}(\pi(\lambda), k)$ equals 1 while the coefficient of $m_{\pi(\lambda)}^{A}$ in $\widetilde{J}_{\lambda}$ equals $v_{\lambda \lambda}\left(k^{-1}\right)$ (cf. (2.6)). Stanley has shown in [30, Theorem 5.6] that the coefficient $v_{\lambda \lambda}\left(k^{-1}\right)$ of $m_{\lambda}$ in $J_{\lambda}$ equals $h_{*}(\lambda)$. Hence the proposition follows.

To our knowledge the result (3.8) has never been stated in this precise form in the literature although it is probably known (cf. [23, p. 316, ex. (5)]). In a sense the two variable case of Jack polynomials was already studied by Koornwinder and Sprinkhuizen-Kuyper in $[14, \S 4]$.

For the product of the ordinary hook-lengths (so $k=1)$ there is the wellknown 'hook-length formula' [19, Chapter I, Ex. 1(4)]. From Proposition 3.3 we obtain a generalization to arbitrary $k$ of this hook-length formula. Let us introduce the notation $a !=\Gamma(a+1)$ and $(b)_{k}=\Gamma(b+k) / \Gamma(b)$ for $a, b, k \in \mathbf{C}$ (so $(b)_{k}$ reduces to the Pochhammer symbol for $\left.k \in \mathbf{Z}^{+}\right)$. Whenever we write $i<j$ we will mean $1 \leq i<j \leq n$.

Corollary 3.4. For any partition $\lambda=\left(\lambda_{1}, \lambda_{2}, \ldots, \lambda_{n}\right)$ we put

$$
\mu_{i}=k(n-i+1)+\lambda_{i}-1 \text { for } i=1,2, \ldots, n .
$$

Then we have for $k \in \mathbf{C}$,

$$
h_{*}(\lambda) / J_{\lambda}\left(1_{n} ; k^{-1}\right)=\mathbf{c}_{A}\left(\pi(\lambda)+\rho_{A}(k), k\right)
$$

or, equivalently,

$$
h_{*}(\lambda)=\frac{k^{-|\lambda|}}{\prod_{i=1}^{n}(k-1) !} \frac{\prod_{i=1}^{n} \mu_{i} !}{\prod_{1 \leq i<j \leq n}\left(\mu_{i}-\mu_{j}\right)_{k}} .
$$

In particular

$$
h_{*}\left(l \omega_{n}\right) / J_{l \omega_{n}}\left(1_{n} ; k^{-1}\right)=1 .
$$

Proof. From [28, Corollary 5.2] one obtains that

$$
P_{A}(\mu, k)(e)=1 / \mathbf{c}_{A}\left(\mu+\rho_{A}(k), k\right)
$$

where $e \in H_{A}$ is the unit element. But $e \in H_{A}$ corresponds to $x=1_{n}$ so that we obtain the corollary from (3.8). To obtain (3.11) we note that $\rho_{A}(k)=$ $\frac{1}{2} k \sum_{l=1}^{n}(n-2 l+1) e_{l}$ and $\left\langle\pi(\lambda), e_{i}-e_{j}\right\rangle=\lambda_{i}-\lambda_{j}$ so that we obtain from (2.29)

$$
\mathbf{c}_{A}\left(\pi(\lambda)+\rho_{A}(k), k\right)=\prod_{i<j} \frac{\Gamma\left(\lambda_{i}-\lambda_{j}+k(j-i)\right) \Gamma(k(j-i+1))}{\Gamma\left(\lambda_{i}-\lambda_{j}+k(j-i+1)\right) \Gamma(k(j-i))} .
$$

(Note that this is a rational function of $k$.) The product

$$
\prod_{i<j} \Gamma(k(j-i+1)) / \Gamma(k(j-i))
$$

reduces to $\prod_{i=1}^{n} \Gamma(i k) / \Gamma(k)$. Moreover, the value of $J_{\lambda}\left(1_{n} ; k^{-1}\right)$ has been determined by Stanley $[30,(2.10)]$ and is given by

$$
J_{\lambda}\left(1_{n} ; k^{-1}\right)=\prod_{(i, j) \in \lambda}\left(n-i+1+k^{-1}(j-1)\right) .
$$


This can be rewritten as

$$
k^{-|\lambda|} \prod_{i=1}^{n}(k(n-i+1))_{\lambda_{i}}=k^{-|\lambda|} \prod_{i=1}^{n} \Gamma\left(k(n-i+1)+\lambda_{i}\right) / \Gamma(k i)
$$

and thus we obtain (3.11). Since $\pi\left(l \omega_{n}\right)=0$ the final result follows from the normalization of the c-function.

Of course it is not hard to get (3.12) directly from (3.6) and (3.13). The case $k=1$ of (3.11) is the hook-length formula in [19]. One can give a direct proof of the 'lower hook-length formula' (3.11) in the following way. Given $\lambda=$ $\left(\lambda_{1}, \ldots, \lambda_{n}\right)$ we consider for $k \in \mathbf{Z}^{+}$the partition $k \cdot \lambda=\left(k \lambda_{1}, \ldots, k \lambda_{n}\right)$. For convenience we assume $l(\lambda)=n$. Let $\lambda^{\prime}=\left(\lambda_{1}^{\prime}, \ldots, \lambda_{m}^{\prime}\right)$ so that $\lambda_{1}^{\prime}=l(\lambda)=n$ and $m=l\left(\lambda^{\prime}\right)=\lambda_{1}$. Then the dual of $k \cdot \lambda$ is $\left(\lambda_{1}^{\prime}, \ldots, \lambda_{1}^{\prime}, \lambda_{2}^{\prime}, \ldots, \lambda_{2}^{\prime}, \lambda_{m}^{\prime}, \ldots\right.$, $\left.\lambda_{m}^{\prime}\right)$ where each $\lambda_{j}^{\prime}$ is repeated $k$ times. If we now use [19, Chapter I, (1.7)] then it follows that the $k m+n$ numbers

$$
k \lambda_{i}+n-i \quad(1 \leq i \leq n), \quad k(j-1)+l+n-\lambda_{j}^{\prime} \quad(1 \leq j \leq m, 0 \leq l \leq k-1)
$$

are a permutation of $0,1,2, \ldots, k m+n-1$. This proves that

$$
\prod_{i=1}^{n}\left(k \lambda_{i}+n-i\right) \prod_{j=2}^{m} \prod_{l=0}^{k-1}\left(n-\lambda_{j}^{\prime}+k(j-1)+l\right)=(k m+n-1) ! /(k-1) !
$$

and hence, with $\lambda$ and $\lambda^{\prime}$ interchanged

$$
\Gamma(k) \prod_{i=1}^{\lambda_{1}}\left(k \lambda_{i}^{\prime}+\lambda_{1}-i\right) \prod_{j=2}^{n}\left(\lambda_{1}-\lambda_{j}+k(j-1)\right)_{k}=\Gamma\left(k n+\lambda_{1}-1\right), \quad k \in \mathbf{Z}^{+},
$$

where $n=\lambda_{1}^{\prime}$. If we apply this successively to the partitions $\left(\lambda_{l}, \lambda_{l+1}, \ldots, \lambda_{n}\right)$ for $l=1,2, \ldots, n$ and multiply the resulting identities then one obtains (3.11) for $k \in \mathbf{Z}^{+}$. Since we are dealing essentially with polynomial identities (3.11) follows for $k \in \mathbf{C}$.

One may ask whether a similar formula holds for the 'upper hook-length' $h^{*}(\lambda)$ as defined in (3.7). The next proposition shows that this is indeed the case.

Proposition 3.5. For any partition $\lambda=\left(\lambda_{1}, \lambda_{2}, \ldots, \lambda_{n}\right)$ we put $\nu_{i}=k(n-i)+\lambda_{i}$ for $i=1,2, \ldots, n$. Then we have for $k \in \mathbf{C}$,

$$
h^{*}(\lambda)=\frac{k^{-|\lambda|} \prod_{i=1}^{n} \nu_{i} !}{\prod_{1 \leq i<j \leq n}\left(\nu_{i}-\nu_{j}-(k-1)\right)_{k}}
$$

or, equivalently,

$$
h^{*}(\lambda) / \prod_{(i, j) \in \lambda}\left(n-i+k^{-1} j\right)=\lim _{\varepsilon \rightarrow 0} \frac{\mathbf{c}_{A}\left(-\pi(\lambda)-\rho_{A}(k)+\varepsilon, k\right)}{\mathbf{c}_{A}\left(-\rho_{A}(k)+\varepsilon, k\right)} .
$$

In particular

$$
h^{*}\left(l \omega_{n}\right) / \prod_{(i, j) \in\left(l^{n}\right)}\left(n-i+k^{-1} j\right)=1
$$


Proof. Let $\lambda$ and $\lambda^{\prime}$ be as above. Note that $\left\{k(j-1)+l+n-\lambda_{j}^{\prime} \mid j=1, l=\right.$ $0,1, \ldots, k-1\}$ is the set of integers $\{0,1, \ldots, k-1\}$. If we delete this set of integers from the $k m+n$ numbers above and subtract $k-1$ from the remaining set then we obtain that the $k(m-1)+n$ numbers

$$
\begin{gathered}
k\left(\lambda_{i}-1\right)+n-i+1 \quad(1 \leq i \leq n), \\
k(j-2)+l+n-\lambda_{j}^{\prime} \quad(2 \leq j \leq m, 1 \leq l \leq k)
\end{gathered}
$$

are a permutation of $1,2, \ldots, k(m-1)+n$. One now proves (3.14) exactly as above for $h_{*}(\lambda)$. To prove (3.15) we first note that

$$
\prod_{i=1}^{n} \nu_{i} !=\prod_{i=1}^{n} \Gamma(k(n-i)+1) \prod_{(i, j) \in \lambda}(k(n-i)+j) .
$$

Since $\prod_{i<j} \Gamma(k(j-i-1)+1+k) / \Gamma(k(j-i-1)+1)$ reduces to $\prod_{i=1}^{n} \Gamma(k(n-i)+1)$ we thus obtain that

$$
\begin{gathered}
\frac{\prod_{i=1}^{n} \nu_{i} !}{\prod_{i<j}\left(\nu_{i}-\nu_{j}-(k-1)\right)_{k}}=\prod_{(i, j) \in \lambda}(k(n-i)+j) \\
\cdot \prod_{i<j} \frac{\Gamma\left(\lambda_{i}-\lambda_{j}+k(j-i-1)+1\right) \Gamma(k(j-i)+1)}{\Gamma(k(j-i-1)+1) \Gamma\left(\lambda_{i}-\lambda_{j}+k(j-i)+1\right)} .
\end{gathered}
$$

Hence from (3.14) we have

$$
h^{*}(\lambda) / \prod_{(i, j) \in \lambda}\left(n-i+k^{-1} j\right)=\prod_{i<j} \frac{(k(j-i-1)+1) \lambda_{i}-\lambda_{j}}{(k(j-i)+1) \lambda_{i}-\lambda_{j}} .
$$

In order to calculate the limit as $\varepsilon \rightarrow 0$ in the $\mathbf{c}_{A}$-functions we may as well consider $\lim _{\varepsilon \rightarrow 0} \mathbf{c}_{A}\left(-\pi(\lambda)-\rho_{A}(k-\varepsilon), k\right) / \mathbf{c}_{A}\left(-\rho_{A}(k-\varepsilon), k\right)$. As in the proof of Corollary 3.4 we have

$$
\begin{aligned}
\lim _{\varepsilon \rightarrow 0} \mathbf{c}_{A}\left(-\pi(\lambda)-\rho_{A}(k-\varepsilon), k\right) / \mathbf{c}_{A}\left(-\rho_{A}(k-\varepsilon), k\right) \\
\quad=\lim _{\varepsilon \rightarrow 0} \prod_{i<j} \frac{\Gamma\left(\lambda_{j}-\lambda_{i}-(k-\varepsilon)(j-i)\right) \Gamma(k-(k-\varepsilon)(j-i))}{\Gamma(-(k-\varepsilon)(j-i)) \Gamma\left(\lambda_{j}-\lambda_{i}-(k-\varepsilon)(j-i)+k\right)} .
\end{aligned}
$$

If we write the right-hand side as $\prod_{i<j} \frac{\left(\lambda_{j}-\lambda_{i}-(k-\varepsilon)(j-i)+k\right)_{\lambda_{i}-\lambda_{j}}}{\left(\lambda_{j}-\lambda_{i}-(k-\varepsilon)(j-i)\right)_{\lambda_{i}-\lambda_{j}}}$ then we can take the limit $\varepsilon \rightarrow 0$. A simple calculation then shows that

$$
\prod_{i<j} \frac{\left(\lambda_{j}-\lambda_{i}-k(j-i)+k\right)_{\lambda_{i}-\lambda_{j}}}{\left(\lambda_{j}-\lambda_{i}-k(j-i)\right)_{\lambda_{i}-\lambda_{j}}}=\prod_{i<j} \frac{(k(j-i-1)+1)_{\lambda_{i}-\lambda_{j}}}{(k(j-i)+1)_{\lambda_{i}-\lambda_{j}}}
$$

which proves $(3.15)$. Since $\pi\left(l \omega_{n}\right)=0$ one immediately obtains (3.16)

Remark 1. To get (3.10) in the same form as (3.15) one can divide (3.10) by $\mathbf{c}_{A}\left(\rho_{A}(k), k\right)$ which equals 1 .

Remark 2. The partition $(n-1, n-2, \ldots, 1,0)$ corresponds under $\pi$ with $\rho_{A}(1) \in P_{A}^{+}$. Hence both partitions $\left(\mu_{1}, \ldots, \mu_{n}\right)$ from Corollary 3.4 and $\left(\nu_{1}, \ldots, \nu_{n}\right)$ from Proposition 3.5 correspond to $\pi(\lambda)+\rho_{A}(k)$. 
The two expressions (3.10) and (3.15) can be used to give the relationship between the two inner products $\langle\cdot, \cdot\rangle_{k}$ in (2.5) and $\langle\cdot, \cdot\rangle_{A_{e}, k}$ in (3.5). Stanley has shown $[30,(3.8)]$ that

$$
\left\langle J_{\lambda}, J_{\lambda}\right\rangle_{k}=h_{*}(\lambda) h^{*}(\lambda)
$$

so that from (3.10) and (3.15) we obtain that

$$
\begin{aligned}
\left\langle J_{\lambda} / h_{*}(\lambda), J_{\lambda} / h_{*}(\lambda)\right\rangle_{k}=h^{*}(\lambda) / h_{*}(\lambda)=\prod_{(i, j) \in \lambda} \frac{n-i+k^{-1} j}{n-i+1+k^{-1}(j-1)} \\
\cdot \mathbf{c}_{A}\left(\pi(\lambda)+\rho_{A}(k), k\right)^{-1} \lim _{\varepsilon \rightarrow 0} \mathbf{c}_{A}\left(-\pi(\lambda)-\rho_{A}(k)+\varepsilon, k\right) / \mathbf{c}_{A}\left(-\rho_{A}(k)+\varepsilon, k\right) .
\end{aligned}
$$

The polynomials $J_{\lambda} / h_{*}(\lambda)$ are denoted by $P_{\lambda}^{\alpha}$ in [20, Chapter VI]. If we now compare this result with [7, Theorem 8.5] (cf. [28, Theorem 2.1]) then it follows that

$$
\begin{aligned}
& \left\langle J_{\lambda} / h_{*}(\lambda), J_{\lambda} / h_{*}(\lambda)\right\rangle_{k} \\
& \quad=\prod_{(i, j) \in \lambda} \frac{n-i+k^{-1} j}{n-i+1+k^{-1}(j-1)} \frac{\left\langle P_{A}(\pi(\lambda), k), P_{A}(\pi(\lambda), k)\right\rangle_{A, k}}{\langle 1,1\rangle_{A, k}}
\end{aligned}
$$

so that from (3.8) and (3.9) we obtain the following result.

Corollary 3.6.

$$
\langle 1,1\rangle_{A_{e}, k} \frac{\left\langle J_{\lambda}, J_{\lambda}\right\rangle_{k}}{\left\langle J_{\lambda}, J_{\lambda}\right\rangle_{A_{e}, k}}=\prod_{(i, j) \in \lambda} \frac{n-i+k^{-1} j}{n-i+1+k^{-1}(j-1)} .
$$

This corollary proves conjecture (C4) in [21, 44 , a result which also follows from the material in [20, Chapter VI, $\S 10]$. The well-known constant $\langle 1,1\rangle_{A_{e}, k}=\int_{T_{B}} \prod_{i<j}\left|x_{i}-x_{j}\right|^{2 k} d x$ is given by $(k n) ! /(k !)^{n}$ ('Dyson's conjecture'; see e.g. [28, §4]).

A straightforward combination of (3.10), (3.15) and (3.17) shows that for arbitrary $\lambda$ one has

$$
\begin{aligned}
& \frac{J_{\lambda}\left(1_{n} ; k^{-1}\right)}{\left\langle J_{\lambda}, J_{\lambda}\right\rangle_{k} k^{|\lambda|}} \prod_{(i, j) \in \lambda}(k(n-i)+j) \\
& \quad=\mathbf{c}_{A}\left(\pi(\lambda)+\rho_{A}(k), k\right)^{-1} \lim _{\varepsilon \rightarrow 0} \frac{\mathbf{c}_{A}\left(-\rho_{A}(k)+\varepsilon, k\right)}{\mathbf{c}_{A}\left(-\pi(\lambda)-\rho_{A}(k)+\varepsilon, k\right)} .
\end{aligned}
$$

For $\lambda=l \omega_{n}$ this result has a nice interpretation in the theory of Jacobi polynomials in $\S 5$ (cf. Corollary 5.3; also see (5.17)).

Finally we mention the following consequence of (3.10), for which we do not have a direct proof. Let us identify $\omega_{n}$ with the partition $(1,1, \ldots, 1)=\left(1^{n}\right)$. For a partition $\lambda=\left(\lambda_{1}, \ldots, \lambda_{n}\right)$ define $\tau(\lambda)=\left(\lambda_{1}-\lambda_{n}, \lambda_{1}-\lambda_{n-1}, \ldots, \lambda_{1}-\right.$ $\left.\lambda_{2}, 0\right)$. From the diagram of $\lambda_{1} \cdot \omega_{n}$ one sees immediately that $\tau\left(\lambda^{\prime}\right)=(\tau(\lambda))^{\prime}$ if $l(\lambda)=n$. Also $\tau^{2}(\lambda)=\lambda-\lambda_{n} \cdot \omega_{n}$. Hence if we define $\tau_{0}(\lambda)=\tau(\lambda)+\lambda_{n} \cdot \omega_{n}$ then $\tau_{0}$ is an involution on partitions. Note that $\pi(\tau(\lambda))=\pi\left(\tau_{0}(\lambda)\right)$ with $\pi$ as always. We now have

Corollary 3.7. Let $\lambda=\left(\lambda_{1}, \ldots, \lambda_{n}\right)$ be a partition. Then

$$
J_{\lambda}\left(1_{n} ; k^{-1}\right) / h_{*}(\lambda)=J_{\tau(\lambda)}\left(1_{n} ; k^{-1}\right) / h_{*}(\tau(\lambda)) .
$$


Proof. Let $w_{0}$ denote the longest Weyl group element in $W_{A} ; w_{0}$ acts as $\left(e_{1}, e_{2}, \ldots, e_{n}\right) \rightarrow\left(e_{n}, e_{n-1}, \ldots, e_{1}\right)$. Then $w_{0} R_{A}^{+}=-R_{A}^{+}$where $-R_{A}^{+}=$ $\left\{-\alpha \mid \alpha \in R_{A}^{+}\right\}$and hence $-w_{0}$ permutes the elements in $R_{A}^{+}$. Since $W_{A}$ acts as orthogonal transformations it follows immediately from the definition of $\mathbf{c}_{\boldsymbol{A}}$ in (2.29) that $\mathbf{c}_{A}\left(-w_{0} \lambda, k\right)=\mathbf{c}_{A}(\lambda, k)$. It is easy to check that $-w_{0} \pi(\lambda)=\pi(\tau(\lambda))$ and since $-w_{0} \rho_{A}(k)=\rho_{A}(k)$ we obtain from (3.10) that $h_{*}(\lambda) / J_{\lambda}\left(1_{n} ; k^{-1}\right)=$ $\mathbf{c}_{A}\left(-w_{0} \pi(\lambda)+\rho_{A}(k), k\right)=h_{*}(\tau(\lambda)) / J_{\tau(\lambda)}\left(1_{n} ; k^{-1}\right)$.

\section{HYPERGEOMETRIC FUNCTIONS}

In this section we will show that the hypergeometric function ${ }_{2} F_{1}$ as defined in (2.10) is a special case of the hypergeometric function associated with root system $B C_{n}$ as defined in [6, $\S 6$ or $\left.7, \S 7\right]$. Recall from Corollary 2.3 that the ${ }_{2} F_{1}$ is an eigenfunction of the operator $\Delta(a, b, c ; k)$ given by (2.13). In the paper [10] by James and Constantine $\Delta(a, b, c ; k)$ for $k=\frac{1}{2}$ and special values of $a, b$ and $c$ occurs, after the change of variables $x_{i}=\cos ^{2} t_{i}(i=1, \ldots, n)$, as the radial part of the Laplace-Beltrami operator on the Grassmann manifold. Since this space has a type $B$ root system it is then clear that for general $a, b, c$ and $k$ the operator $\Delta(a, b, c ; k)$ is the generalized radial part of the LaplaceBeltrami operator associated with root system $B C_{n}$ in the sense of $\S 2$. This result was also noted by Korányi in [15, §4]. We now describe explicitly how to interpret $\Delta(a, b, c ; k)$ as a generalized radial part.

For $i=1, \ldots, n$ we put $y_{i}=\cosh t_{i}\left(t_{i} \in \mathbf{C}\right)$ and we let $z_{j}$ be the $j$ th elementary symmetric polynomial in $y_{1}, \ldots, y_{n}$ for $j=1, \ldots, n$. Furthermore we let

$$
x_{i}=\frac{1}{2}\left(1-y_{i}\right)=\frac{1}{2}-\frac{1}{4}\left(e^{t_{i}}+e^{-t_{i}}\right)=-\sinh ^{2} \frac{1}{2} t_{i}, \quad i=1, \ldots, n .
$$

Finally we let $w_{j}$ denote the $j$ th elementary symmetric polynomial in the $x_{i}$. (Recall from $\S 2 \mathrm{~b}$ that $e^{t_{i}}=e^{e_{i}}(\dot{t})$ for $t=\left(t_{1}, \ldots, t_{n}\right) \in \mathbf{C}^{n}$.)

From (4.1) it is clear that from a symmetric function in the variables $x_{i}$ one obtains a $W_{B}$-invariant function on $H_{B}$. In this sense we can consider the function ${ }_{2} F_{1}\left(a, b ; c ; x ; k^{-1}\right)$ as a $W_{B}$-invariant function on $H_{B}$. A straightforward calculation shows that under the change of coordinates (4.1) the operator $\Delta(a, b, c ; k)$ becomes

$$
\begin{aligned}
-\Delta(a, b, c ; k)= & \sum_{i=1}^{n} \partial_{t_{i}}^{2}+k \sum_{i<j}\left(\operatorname{coth} \frac{1}{2}\left(t_{i}+t_{j}\right)\left(\partial_{t_{i}}+\partial_{t_{j}}\right)\right. \\
& \left.+\operatorname{coth} \frac{1}{2}\left(t_{i}-t_{j}\right)\left(\partial_{t_{i}}-\partial_{t_{j}}\right)\right) \\
& +\sum_{i=1}^{n}\left((2 a+2 b+1-2 c) \operatorname{coth} t_{i} \partial_{t_{i}}\right. \\
& \left.+(2 c-a-b-1-k(n-1)) \operatorname{coth} \frac{1}{2} t_{i} \partial\right) .
\end{aligned}
$$

If we compare this with (2.28) and recall the notation $\left(k_{1}, k_{2}, k_{3}\right)$ in (2.22) then we see that

$$
\Delta(a, b, c ; k)=-L_{B}\left(k_{1}, k_{2}, k_{3}\right)
$$


with

$$
\begin{aligned}
& k_{1}=2 c-a-b-1-k(n-1), \\
& k_{2}=a+b+\frac{1}{2}-c, \quad k_{3}=k .
\end{aligned}
$$

For later use we note that

$$
k_{1}+2 k_{2}+k_{3}(n-1)=a+b .
$$

There are many places in the literature where the operator $L_{B}\left(k_{1}, k_{2}, k_{3}\right)$ is defined by (4.2) and then calculated in the $y$-coordinates. For some more references see $[1, \S 7]$ where the operator is also given in the $z$-coordinates.

Now let $l \in \mathbf{Z}^{+}$and consider ${ }_{2} F_{1}\left(-l, b ; c ; x ; k^{-1}\right)$. Since

$$
(-l)_{\lambda}=(-l)_{\lambda_{1}}(-l-k)_{\lambda_{2}} \cdots(-l-k(n-1))_{\lambda_{n}}
$$

and $(-l)_{\lambda_{1}}=0$ if $\lambda_{1} \geq l+1$ we see that

$$
{ }_{2} F_{1}\left(-l, b ; c ; x ; k^{-1}\right)=\sum_{\lambda} \frac{(-l)_{\lambda}(b)_{\lambda}}{(c)_{\lambda} k^{|\lambda|}}\left\langle J_{\lambda}, J_{\lambda}\right\rangle^{-1} J_{\lambda}\left(x ; k^{-1}\right)
$$

is a polynomial. In (4.7) the sum is taken over all partitions $\lambda=\left(\lambda_{1}, \ldots, \lambda_{n}\right)$ such that $l \geq \lambda_{1} \geq \lambda_{2} \geq \cdots \geq \lambda_{n} \geq 0$. This is equivalent to $\lambda \subset\left(l^{n}\right)=$ $(l, l, \ldots, l)$. Note that the partition $\left(l^{n}\right)$ corresponds to the weight $l \omega_{n} \in P_{B}^{+}$ with $\omega_{n}$ as in (2.16).

To formulate the next lemma we need some notations and results concerning $W_{B}$-invariant differential operators. In [3, Chapter II, $\S 2, \S \S a$ and b] it was shown by explicit construction that $L_{B}\left(k_{1}, k_{2}, k_{3}\right)$ is contained in an $n$ dimensional commutative algebra $\mathscr{D}$ of $W_{B}$-invariant differential operators with rational functions in the $y_{i}$ as coefficients. For special values of the parameters $k_{1}, k_{2}, k_{3}$ this follows from the well-known fact that the algebra of invariant differential operators on a Riemannian symmetric space is commutative (cf. [6, Remark 2.11]). In [27, Theorem 3.6.a] it is shown that such a result holds for arbitrary root systems and arbitrary parameters $(\mathscr{D}$ is denoted by $\mathscr{S}(0, \kappa)$ in [27]). In any case one obtains that the so-called Harish-Chandra homomorphism $\gamma: \mathscr{D} \rightarrow \mathbf{C}\left[t_{1}, \ldots, t_{n}\right]^{W_{B}}$ is an algebra isomorphism for all values of $\left(k_{1}, k_{2}, k_{3}\right)$. Here $\mathbf{C}\left[t_{1}, \ldots, t_{n}\right]^{W_{B}}$ is the algebra of $W_{B}$-invariant polynomials on $\mathbf{C}^{n}$. One can describe $\gamma$ as follows. Let $\mathbf{C}[\mathscr{K}]$ denote the algebra of polynomials in $k_{1}, k_{2}, k_{3}$ and $\mathbf{A}_{n}$ the Weyl algebra of differential operators in the variables $z_{j}(j=1, \ldots, n)$ with polynomials in the $z_{j}$ as coefficients. Then we let $\mathscr{D}$ be the algebra consisting of those $D \in \mathbf{C}[\mathscr{K}] \otimes \mathbf{A}_{n}$ that commute with $L_{B}\left(k_{1}, k_{2}, k_{3}\right)$. The Jacobi polynomial $P_{B}(\lambda ; \kappa)$ then satisfies

$$
D \phi=\gamma(D)\left(\lambda+\rho_{B}(\kappa)\right) \phi, \quad D \in \mathscr{D},
$$

for some $\gamma(D) \in \mathbf{C}[\mathscr{K}] \otimes \mathbf{C}\left[t_{1}, \ldots, t_{n}\right]^{W_{B}}$. For more details we refer to [6, $\S 2$ and $27, \S \S 2-3]$.

Proposition 4.1. If $l \in \mathbf{Z}^{+}$then ${ }_{2} F_{1}\left(-l, b ; c ; x ; k^{-1}\right)$ considered as function on $H_{B}$ under the change of coordinates (4.1) satisfies

$$
L_{B}(\kappa)_{2} F_{1}=\left\langle l \omega_{n}, l \omega_{n}+2 \rho_{B}(\kappa)\right\rangle_{2} F_{1},
$$

where $\kappa=\left(k_{1}, k_{2}, k_{3}\right)$ is related to $(b, c, k)$ as in (4.4) with $a=-l$. Furthermore

$$
{ }_{2} F_{1}\left(-l, b ; c ; x ; k^{-1}\right)=\mathbf{c}_{B}\left(l \omega_{n}+\rho_{B}(\kappa), \kappa\right) P_{B}\left(l \omega_{n}, \kappa\right)(\dot{t})
$$


In particular ${ }_{2} F_{1}\left(-l, b ; c ; x ; k^{-1}\right)$ satisfies (4.8) for all $D \in \mathscr{D}$.

Proof. For $\lambda=l \omega_{n}=\left(l^{n}\right)$ we get from (2.30) that

$$
\left\langle\lambda, \lambda+2 \rho_{B}(\kappa)\right\rangle=n l\left(k_{1}+2 k_{2}+k_{3}(n-1)+l\right)=-n a b,
$$

where the last equality follows from (4.5) with $a=-l$. The first statement of the proposition now follows immediately from (4.3) and (2.12). From (4.7) and (2.6) we obtain

$$
{ }_{2} F_{1}\left(-l, b ; c ; x ; k^{-1}\right)=\sum_{\lambda \subset\left(l^{n}\right)} \sum_{\nu \leq \lambda} \frac{(-l)_{\lambda}(b)_{\lambda}}{(c)_{\lambda} k^{|\lambda|}} \frac{v_{\lambda \nu}\left(k^{-1}\right)}{\left\langle J_{\lambda}, J_{\lambda}\right\rangle} m_{\nu}(x)
$$

where $m_{\nu}(x)=\sum_{\alpha \in S_{n} \cdot \nu} x^{\alpha}$. The support of this polynomial in $x_{1}, \ldots, x_{n}$, i.e. those $\alpha=\left(\alpha_{1}, \ldots, \alpha_{n}\right)$ for which the coefficient of $x^{\alpha}$ is nonzero, is contained in the set $\left\{\left(\alpha_{1}, \ldots, \alpha_{n}\right) \mid 0 \leq \alpha_{i} \leq l\right\}$, since $\nu \leq\left(l^{n}\right)$ implies $\nu_{i} \leq l$ for all $i$. Applying the transformation $x_{i}=\frac{1}{2}-\frac{1}{4}\left(e^{t_{i}}+e^{-t_{i}}\right)$ will result in a $W_{B}$-invariant polynomial on $H_{B}$ with its support contained in $\left\{\left(\alpha_{1}, \ldots, \alpha_{n}\right)|| \alpha_{i} \mid \leq l\right\}$. Considered as vectors in $\mathbf{R}^{n}$ this set is precisely $\Pi_{B}\left(l \omega_{n}\right)$. Hence ${ }_{2} F_{1}\left(-l, b ; c ; x ; k^{-1}\right)$ is a $W_{B}$-invariant polynomial of the form

$$
\sum_{\nu \in \Pi_{B}\left(l \omega_{n}\right)} d(\nu, b, c, l, k) e^{\nu}
$$

for certain constants $d(\nu, b, c, l, k)$. As we have seen above, it is also an eigenfunction of $L_{B}(\kappa)$. As stated in $\S 2 \mathrm{~b}$ these two properties characterize the Jacobi polynomials $P_{B}\left(l \omega_{n}, \kappa\right)$ up to normalization. From [28, Corollary 5.2] we obtain that $P_{B}(\lambda, \kappa)(e)=\mathrm{c}_{B}\left(\lambda+\rho_{B}(\kappa), \kappa\right)^{-1}$ where $e \in H_{B}$ is the unit element. Since $J_{\lambda}$ is a homogeneous polynomial of degree $|\lambda|$ it follows from the definition of the ${ }_{2} F_{1}$ that ${ }_{2} F_{1}\left(-l, b ; c ; 0 ; k^{-1}\right)=1$. Since $e \in H_{B}$ corresponds to $x=0$ the second statement in the proposition follows. The final statement is a standard result using the form of the operators in $\mathscr{D}$ and the commutativity of $\mathscr{D}$ (cf. [6, p. 341]). This completes the proof.

In Proposition 4.3 we will show that $\mathbf{c}_{B}\left(l \omega_{n}+\rho_{B}(\kappa), \kappa\right)=2^{-2 n l}(b)_{\left(l^{n}\right)} /(c)_{\left(l^{n}\right)}$. Now let $F_{B}(\lambda, \kappa ; h)$ denote the hypergeometric function associated with the root system $B C_{n}$ as defined in [6, $\S 6$ or $\left.7, \S 7\right]$. Here $\lambda \in \mathbf{C}^{n}, \kappa \in \mathscr{K}$ and $h \in H_{B}$. Recall from [6 or 7] that for $\lambda \in P_{B}^{+}$one has

$$
F_{B}\left(-\left(\lambda+\rho_{B}(\kappa)\right), \kappa ; \dot{t}\right)=\mathbf{c}_{B}\left(\lambda+\rho_{B}(\kappa), \kappa\right) P_{B}(\lambda, \kappa)(\dot{t}), \quad t \in \mathbf{C}^{n} .
$$

(One can ignore the sign in $F_{B}$ since $w_{0}=-1 \in W_{B}$ and $F_{B}$ is $W_{B}$-invariant. Also see the remark after the definition of the Jacobi polynomials.)

Theorem 4.2. Let ${ }_{2} F_{1}\left(a, b ; c ; x ; k^{-1}\right)$ be defined by $(2.10)$ and let $F_{B}(\lambda, \kappa ; h)$ be the hypergeometric function associated with the root system $B C_{n}$. Then

$$
{ }_{2} F_{1}\left(a, b ; c ; x ; k^{-1}\right)=F_{B}\left(-\left(\lambda+\rho_{B}(\kappa)\right), \kappa ; \dot{t}\right)
$$

where $x=\left(x_{1}, \ldots, x_{n}\right), \kappa=\left(k_{1}, k_{2}, k_{3}\right), t=\left(t_{1}, \ldots, t_{n}\right) \in \mathbf{C}^{n}$ and

$$
\begin{aligned}
x_{i} & =\frac{1}{2}-\frac{1}{4}\left(e^{t_{i}}+e^{-t_{i}}\right), \quad i=1, \ldots, n, \\
\lambda & =-a \omega_{n}, \quad a+b=k_{1}+2 k_{2}+k_{3}(n-1), \\
c & =k_{1}+k_{2}+k_{3}(n-1)+\frac{1}{2}, \quad k=k_{3} .
\end{aligned}
$$


Proof. We first want to prove that for general parameter $a$ the function ${ }_{2} F_{1}$, considered as a $W_{B}$-invariant function under the change of coordinates (4.1), satisfies the system of equations (4.8). As was shown in [6, §2], $D$ as differential operator in the $z$-coordinates has polynomial coefficients (our coordinates $z_{1}, \ldots, z_{n}$ are indeed the $z$-coordinates for this case as defined in $\left.[6, \S 2]\right)$. Hence $D$ as differential operator in the $w$-coordinates has polynomials coefficients. Moreover, the $J_{\lambda}\left(x ; k^{-1}\right)$ can be expressed as weighted homogeneous polynomials in the $w_{j}$ since the $w_{j}$ are the $j$ th elementary symmetric polynomials in the $x_{i}$. By 'weighted homogeneous' we mean that we take the degree of $w_{j}$ to be $j$. When we consider (4.8) in the $w$-coordinates it can now be separated into its weighted homogeneous parts. In each of the resulting equations the parameter $a$ occurs polynomially. Since we have shown in Proposition 4.1 that the ${ }_{2} F_{1}$ satisfies (4.8) for $a=-l$ with $l \in \mathbf{Z}^{+}$we can conclude that the ${ }_{2} F_{1}$ satisfies $(4.8)$ for all $a$. Since ${ }_{2} F_{1}$ is analytic in $\left|x_{i}\right|<1$ we obtain from [6, Theorem 6.9] that

$$
{ }_{2} F_{1}\left(a, b ; c ; x ; k^{-1}\right)=d(\lambda, \kappa) F_{B}\left(-\left(\lambda+\rho_{B}(\kappa)\right), \kappa ; \dot{t}\right)
$$

where $(a, b, c, k, x)$ correspond to $(\lambda, \kappa, t)$ as stated in the theorem (cf. (4.4) and Proposition 4.1) and $d(\lambda, \kappa)$ is a constant depending meromorphically on $\lambda$ and $\kappa$. It remains to show that $d(\lambda, \kappa)=1$. Now Conjecture 6.11 in [6] states that (for arbitrary root systems and arbitrary $\lambda) F_{B}(\lambda, \kappa ; e)=1$ where $e \in H_{B}$ is the unit element. As in Proposition 4.1 it would then follow that $d(\lambda, \kappa)=1$. Although the conjecture has recently been established by Opdam [29] we will not use this result and present an alternative proof instead. First of all, just as in the group-cases, it follows from the construction of the hypergeometric function (which is the spherical function in the group-case) that

$$
\lim _{t \rightarrow-\infty} e^{\mu-\rho_{B}(\kappa)}\left(h_{t}\right) F_{B}\left(-\mu, \kappa ; h_{t}\right)=\mathbf{c}_{B}(\mu, \kappa)
$$

where $h_{t}=(t v)^{\bullet}$ with $t \in \mathbf{R}$ and $v=\left(v_{1}, \ldots, v_{n}\right)$ and $\mu$ in the positive Weyl chamber of $B C_{n}$. If we take $\mu=-a \omega_{n}+\rho_{B}(\kappa)$ with $\operatorname{Re} a<0$ then it follows that

$$
\lim _{t \rightarrow-\infty} e^{-a \omega_{n}}\left(h_{t}\right) F_{B}\left(a \omega_{n}-\rho_{B}(\kappa), \kappa ; h_{t}\right)=\mathbf{c}_{B}\left(-a \omega_{n}+\rho_{B}(\kappa), \kappa\right) .
$$

Now use the Kummer relation (2.14) for the ${ }_{2} F_{1}$, multiply (4.12) by $e^{-a \omega_{n}}\left(h_{t}\right)$ and let $t \rightarrow-\infty$. Then one obtains that

$$
\begin{array}{r}
d(\lambda, \kappa) \mathbf{c}_{B}\left(\lambda+\rho_{B}(\kappa), \kappa\right)=\lim _{t \rightarrow-\infty} e^{-a \omega_{n}}\left(h_{t}\right) \prod_{i=1}^{n}\left(1-x_{i}\right)^{-a} \\
\cdot{ }_{2} F_{1}\left(c-b, a ; c ; x_{1} /\left(x_{1}-1\right), \ldots, x_{n} /\left(x_{n}-1\right) ; k^{-1}\right)
\end{array}
$$

where $(a, b, c, k, x)$ correspond to $(\lambda, \kappa, t)$ as above. In particular $x_{i} /\left(x_{i}-1\right)$ tends to 1 as $t \rightarrow-\infty$. Also $1-x_{i}=\frac{1}{2}+\frac{1}{4}\left(e^{v_{i} t}+e^{-v_{i} t}\right)$ with $v_{i}>0$ for all $i$ so that $e^{-a \omega_{n}}\left(h_{t}\right) \prod_{i=1}^{n}\left(1-x_{i}\right)^{-a}$ tends to $4^{n a}$ as $t \rightarrow-\infty$. Hence it follows that

$$
d(\lambda, \kappa) \mathbf{c}_{B}\left(\lambda+\rho_{B}(\kappa), \kappa\right)=2^{2 n a}{ }_{2} F_{1}\left(c-b, a ; c ; 1_{n} ; k^{-1}\right) .
$$

The Gauss summation formula (2.15) then implies that

$$
d(\lambda, \kappa) \mathbf{c}_{B}\left(\lambda+\rho_{B}(\kappa), \kappa\right)=2^{2 n a} \prod_{i=1}^{n} \frac{\Gamma(c-k(i-1)) \Gamma(b-a-k(i-1))}{\Gamma(b-k(i-1)) \Gamma(c-a-k(i-1))}
$$


under the appropriate conditions. The result $d(\lambda, \kappa)=1$-and hence the theorem-now follows from Proposition 4.3 below and analytic continuation in $\lambda$ and $\kappa$.

Proposition 4.3. With $\kappa=\left(k_{1}, k_{2}, k_{3}\right)$ we have

$$
\mathbf{c}_{B}\left(-a \omega_{n}+\rho_{B}(\kappa), \kappa\right)=2^{2 n a} \prod_{i=1}^{n} \frac{\Gamma(c-k(i-1)) \Gamma(b-a-k(i-1))}{\Gamma(b-k(i-1)) \Gamma(c-a-k(i-1))}
$$

where $c=k_{1}+k_{2}+\frac{1}{2}+k_{3}(n-1), b=k_{1}+2 k_{2}+k_{3}(n-1)-a$ and $k=k_{3}$. In particular one has for $-a=l \in \mathbf{Z}^{+}$that

$$
\mathbf{c}_{B}\left(l \omega_{n}+\rho_{B}(\kappa), \kappa\right)=2^{-2 n l}(b)_{\left(l^{n}\right)} /(c)_{\left(l^{n}\right)} .
$$

Proof. Note that $R_{B}=\left\{\alpha^{\vee} \mid \alpha \in R_{B}\right\}$ and that $\left\langle a \omega_{n}, e_{i}-e_{j}\right\rangle=0$ so that with $\kappa=\left(k_{1}, k_{2}, k_{3}\right)$ as in (2.22) one obtains from the definition (2.29) of the c-function and the explicit expression of $\rho_{B}(\kappa)$ in (2.30) that

$$
\begin{gathered}
\mathbf{c}_{B}\left(-a \omega_{n}+\rho_{B}(\kappa), \kappa\right)=\prod_{i=1}^{n} \frac{\Gamma\left(k_{1}+2 k_{2}+2 k_{3}(n-i)-2 a\right) \Gamma\left(2 k_{1}+2 k_{2}+2 k_{3}(n-i)\right)}{\Gamma\left(k_{1}+2 k_{2}+2 k_{3}(n-i)\right) \Gamma\left(2 k_{1}+2 k_{2}+2 k_{3}(n-i)-2 a\right)} \\
\cdot \frac{\Gamma\left(k_{1}+k_{2}+k_{3}(n-i)-a\right) \Gamma\left(k_{1}+2 k_{2}+k_{3}(n-i)\right)}{\Gamma\left(k_{1}+k_{2}+k_{3}(n-i)\right) \Gamma\left(k_{1}+2 k_{2}+k_{3}(n-i)-a\right)} \\
\cdot \prod_{1 \leq i<j \leq n} \frac{\Gamma\left(k_{1}+2 k_{2}+k_{3}(2 n-i-j)-2 a\right) \Gamma\left(k_{1}+2 k_{2}+k_{3}(2 n-i-j+1)\right)}{\Gamma\left(k_{1}+2 k_{2}+k_{3}(2 n-i-j)\right) \Gamma\left(k_{1}+2 k_{2}+k_{3}(2 n-i-j+1)-2 a\right)} .
\end{gathered}
$$

In the products of the form $\prod_{i<j} \frac{\Gamma(r+s(2 n-i-j))}{\Gamma(r+s(2 n-i-j+1))}$ there is a lot of cancellation in successive quotients and only $\prod_{i=1}^{n-1} \frac{\Gamma(r+s(n-i))}{\Gamma(r+2 s(n-i))}$ remains. If we substitute this into our expression for the c-function then we obtain

$$
\begin{gathered}
\mathbf{c}_{B}\left(-a \omega_{n}+\rho_{B}(\kappa), \kappa\right)=\prod_{i=1}^{n} \frac{\Gamma\left(2 k_{1}+2 k_{2}+2 k_{3}(n-i)\right)}{\Gamma\left(2 k_{1}+2 k_{2}+2 k_{3}(n-i)-2 a\right)} \\
. \frac{\Gamma\left(k_{1}+k_{2}+k_{3}(n-i)-a\right) \Gamma\left(k_{1}+2 k_{2}+k_{3}(n-i)-2 a\right)}{\Gamma\left(k_{1}+k_{2}+k_{3}(n-i)\right) \Gamma\left(k_{1}+2 k_{2}+k_{3}(n-i)-a\right)} .
\end{gathered}
$$

If we put $z_{1}=k_{1}+k_{2}+k_{3}(n-i)$ and $z_{2}=z_{1}-a$ and use the duplication formula for the $\Gamma$-function then it follows that

$$
\mathbf{c}_{B}\left(-a \omega_{n}+\rho_{B}(\kappa), \kappa\right)=\prod_{i=1}^{n} \frac{2^{2 z_{1}} \Gamma\left(z_{1}+\frac{1}{2}\right) \Gamma\left(z_{2}+k_{2}-a\right)}{2^{2 z_{2}} \Gamma\left(z_{2}+\frac{1}{2}\right) \Gamma\left(z_{2}+k_{2}\right)}
$$

which proves the proposition since $2 z_{1}-2 z_{2}=2 a, z_{1}+\frac{1}{2}=c-k(i-1)$ and $z_{2}+k_{2}=b-k(i-1)$.

The case $n=2$ of Theorem 4.2 has essentially been established by Yan [32, Proposition 7] and [33, Proposition 6.5].

Note that Theorem 4.2 proves in particular that $F_{B}\left(a \omega_{n}-\rho_{B}(\kappa), \kappa ; e\right)=1$ since ${ }_{2} F_{1}\left(a, b ; c ; 0 ; k^{-1}\right)=1$.

\section{JACOBI POLYNOMIALS}

In this section we will treat the Jacobi polynomials and in particular we will show that the Jacobi polynomials as defined by Herz, James and Constantine 
and, more recently Macdonald coincide with Jacobi polynomials associated with $B C_{n}$.

Let us recall that in the one-variable case the Jacobi polynomials $P_{l}^{(\alpha, \beta)}(x)$ can be defined on the interval $[-1,1]$ by

$$
P_{l}^{(\alpha, \beta)}(x)=\frac{(\alpha+1)_{l}}{l !}{ }_{2} F_{1}\left(-l, l+\alpha+\beta+1 ; \alpha+1 ; \frac{1}{2}(1-x)\right) .
$$

It seems natural to conjecture that a nice class of orthogonal polynomials in several variables can be obtained from the ${ }_{2} F_{1}\left(a, b ; c ; x ; k^{-1}\right)$ as defined in (2.10). Indeed, for $k=\frac{1}{2}$ Herz $[8, \S 6]$ defines Jacobi polynomials in $n$ variables $x=\left(x_{1}, \ldots, x_{n}\right)$ by

$$
\begin{aligned}
P_{l}^{(\alpha, \beta)}(x)= & \prod_{i=1}^{n} \frac{\Gamma(\alpha+l+1+(i-1) / 2)}{\Gamma(\alpha-(i-1) / 2)} \\
& \cdot{ }_{2} F_{1}\left(-l,-l+\alpha+\beta+\frac{1}{2}(n+1) ; \alpha+\frac{1}{2}(n+1) ; x ; 2\right) .
\end{aligned}
$$

As Herz remarks, for $n>1$ this set of polynomials is "obviously incomplete" and he wonders how to define a complete set of Jacobi polynomials. This problem was picked up again by James and Constantine [10]. For any partition $\lambda$ they define generalized Jacobi polynomials $P_{\lambda}$ associated with the Grassmann manifold as an expansion of the form

$$
P_{\lambda}(x)=\sum_{\mu} u_{\lambda \mu} J_{\mu}(x ; 2)
$$

for certain (undetermined) coefficients $u_{\lambda \mu}[10,(14.1)]$. Then they use the radial part of the Laplace-Beltrami operator on the Grassmann manifold to find a recurrence relation for the coefficients $u_{\lambda \mu}$ [10, Theorem 15.1]. For the special case $\lambda=\left(l^{n}\right)$ they give $P_{\left(l^{n}\right)}$ explicitly [10, (15.4)] but they do not mention any relation with the Herz Jacobi polynomials as given by (5.2). Macdonald's development of Jacobi polynomials for general $k$ in [24] follows that of [10]. Let us describe the results of [24] here.

Let $E(\alpha, \beta, k)$ be the differential operator

$$
\begin{aligned}
-E(\alpha, \beta, k)= & \sum_{i=1}^{n} x_{i}\left(1-x_{i}\right) \partial_{x_{i}}^{2}+2 k \sum_{i, j=1 ; i \neq j}^{n} \frac{x_{i}\left(1-x_{i}\right)}{\left(x_{i}-x_{j}\right)} \partial_{x_{i}} \\
& +\sum_{i=1}^{n}\left(\alpha+1-(\alpha+\beta+2) x_{i}\right) \partial_{x_{i}} .
\end{aligned}
$$

For $\alpha, \beta>0$ and any partition $\lambda$ define the Jacobi polynomial $G_{\lambda}^{(\alpha, \beta)}\left(x ; k^{-1}\right)$ in the $n$ variables $x=\left(x_{1}, \ldots, x_{n}\right)$ as the polynomial of the form

$$
G_{\lambda}^{(\alpha, \beta)}\left(x ; k^{-1}\right)=\sum_{\mu \subset \lambda} u_{\lambda \mu}(\alpha, \beta, k) \Omega_{\mu}\left(x ; k^{-1}\right)
$$

that satisfies

$$
\begin{aligned}
& E(\alpha, \beta, k) G_{\lambda}^{(\alpha, \beta)}\left(x ; k^{-1}\right) \\
& \quad=\left((\alpha+\beta+2+2 k(n-1))|\lambda|+2\left(n\left(\lambda^{\prime}\right)-k n(\lambda)\right)\right) G_{\lambda}^{(\alpha, \beta)}\left(x ; k^{-1}\right),
\end{aligned}
$$

where $\Omega_{\lambda}$ and $n(\lambda)$ are given by $(2.8)$ and (2.2) respectively. The $G_{\lambda}^{(\alpha, \beta)}\left(x ; k^{-1}\right)$ are normalized by the requirement that the coefficient $u_{\lambda \lambda}$ of $\Omega_{\lambda}$ in (5.3) equals 
$(-1)^{|\lambda|}$. It is now crucial that one can formulate (5.4) in terms of the root system $B C_{n}$. This is suggested not only by the case $k=\frac{1}{2}$ in [10] but also by the fact that after the change of variables $x_{i}=\frac{1}{2}\left(1-y_{i}\right)$ one obtains from $E(\alpha, \beta, k)$ the operator $-\frac{1}{4} D^{\alpha, \beta, \gamma}$ with $\gamma=k-\frac{1}{2}$ that was defined by Vretare $[31$, p. 816] (Vretare also defined Jacobi polynomials and showed that these were eigenfunctions of $D^{\alpha, \beta, \gamma}$; also see [3]). We have

$$
E(\alpha, \beta, k)=-\Delta(a, b, c ; k)
$$

where

$$
c=\alpha+1+k(n-1), \quad a+b=\alpha+\beta+1+k(n-1)
$$

(this explains why we choose to use $k^{-1}$ instead of $\alpha$ in the theory of Jack polynomials). Note that for $k=\frac{1}{2}$ and $a=-l$ one obtains the parameters in (5.2) and that for $n=1$ this reduces to the familiar parameter change in the classical Gauss hypergeometric function in (5.1). Often the parameter $p=k(n-1)+1$ is introduced (e.g. in [8], where $k=\frac{1}{2}$ so that $p=\frac{1}{2}(n+1)$, and also in [24]). If we combine (4.3) with (5.5) and also (4.4) with (5.6) then we obtain that

$$
E(\alpha, \beta, k)=L_{B}\left(k_{1}, k_{2}, k_{3}\right)
$$

where

$$
k_{1}=\alpha-\beta, \quad k_{2}=\beta+\frac{1}{2}, \quad k_{3}=k .
$$

If we put $\gamma=k-\frac{1}{2}=k_{3}-\frac{1}{2}$ then the transformation $(\alpha, \beta, \gamma) \leftrightarrow\left(k_{1}, k_{2}, k_{3}\right)$ given by (5.8) is very well known in the literature on Jacobi polynomials associated with $B C_{n}$ (see e.g. [1, §7] and the references given there). As for the eigenvalue in (5.4) we note that for $\lambda=\left(\lambda_{1}, \ldots, \lambda_{n}\right)$, which we identify as usual with $\lambda_{1} e_{1}+\cdots+\lambda_{n} e_{n}$, we have by $(2.30)$

$$
\begin{aligned}
\left\langle\lambda, \lambda+2 \rho_{B}(\kappa)\right\rangle= & \left(k_{1}+2 k_{2}\right)|\lambda|+2 k_{3} \sum_{i=1}^{n} \lambda_{i}(n-i)+\sum_{i=1}^{n} \lambda_{i}^{2} \\
= & \left(k_{1}+2 k_{2}+2 k_{3}(n-1)+1\right)|\lambda| \\
& -2 k_{3} \sum_{i=1}^{n} \lambda_{i}(i-1)+\sum_{i=1}^{n} \lambda_{i}\left(\lambda_{i}-1\right) .
\end{aligned}
$$

From the definition of $n(\lambda)$ in (2.2) and the relations (4.4) then follows that

$$
\begin{aligned}
\left\langle\lambda, \lambda+2 \rho_{B}(\kappa)\right\rangle & =\left(k_{1}+2 k_{2}+2 k_{3}(n-1)+1\right)|\lambda|+2\left(n\left(\lambda^{\prime}\right)-k_{3} n(\lambda)\right) \\
& =(a+b+k(n-1)+1)|\lambda|+2\left(n\left(\lambda^{\prime}\right)-k n(\lambda)\right) .
\end{aligned}
$$

Hence by (5.6)

$$
\left\langle\lambda, \lambda+2 \rho_{B}(\kappa)\right\rangle=(\alpha+\beta+2+2 k(n-1))|\lambda|+2\left(n\left(\lambda^{\prime}\right)-k n(\lambda)\right)
$$

so that with (5.7) we can reformulate (5.4) in terms of the root system $B C_{n}$ as follows

$$
L_{B}(\kappa) G_{\lambda}^{(\alpha, \beta)}\left(x ; k^{-1}\right)=\left\langle\lambda, \lambda+2 \rho_{B}(\kappa)\right\rangle G_{\lambda}^{(\alpha, \beta)}\left(x ; k^{-1}\right) .
$$

Here $\kappa=\left(k_{1}, k_{2}, k_{3}\right)$ is related to $(\alpha, \beta, k)$ as in (5.8).

Recall from $\S 2 \mathrm{~b}$ the Jacobi polynomials $P_{B}(\lambda, \kappa)$ associated with root system $B C_{n}$ and from $\S 4$ the change of coordinates (4.1). Also recall the definition of $h_{*}(\lambda)$ in (3.6) and the explicit value of $J_{\lambda}\left(1_{n} ; k^{-1}\right)$ from (3.13). The proof of the next theorem is similar to the one given for Proposition 4.1. 
Theorem 5.1. For any partition $\lambda$ one has

$$
G_{\lambda}^{(\alpha, \beta)}\left(x ; k^{-1}\right)=\frac{2^{-2|\lambda|} h_{*}(\lambda)}{J_{\lambda}\left(1_{n} ; k^{-1}\right)} P_{B}(\lambda, \kappa)(\dot{t})
$$

where $\kappa=\left(k_{1}, k_{2}, k_{3}\right)=\left(\alpha-\beta, \beta+\frac{1}{2}, k\right)$ and $x_{i}=\frac{1}{2}-\frac{1}{4}\left(e^{e_{i}}+e^{-e_{i}}\right)(\dot{t})$ for $i=1, \ldots, n$.

Proof. From (5.3) and the definition of $\Omega_{\lambda}$ (and $J_{\lambda}$ ) follows that

$$
G_{\lambda}^{(\alpha, \beta)}\left(x ; k^{-1}\right)=\sum_{\mu \subset \lambda} \sum_{\nu \leq \mu} \frac{u_{\lambda \mu} v_{\nu \mu}\left(k^{-1}\right)}{J_{\mu}\left(1_{n} ; k^{-1}\right)} m_{\nu}(x) .
$$

Note that the coefficient of $m_{\lambda}$ equals $u_{\lambda \lambda} v_{\lambda \lambda}\left(k^{-1}\right) / J_{\lambda}\left(1_{n} ; k^{-1}\right)$. As we have seen in Proposition 3.2 the set

$$
\text { \{partitions } \nu \mid \exists \text { partition } \mu \text { with } \nu \leq \mu, \mu \subset \lambda \text { \} }
$$

coincides with the intersection of $\Pi_{B}(\lambda)$ with the positive Weyl chamber of $B C_{n}$. Let us denote this set by $\Pi_{B}^{+}(\lambda)$ so that

$$
G_{\lambda}^{(\alpha, \beta)}\left(x ; k^{-1}\right)=\sum_{\nu \in \Pi_{B}^{+}(\lambda)} d(\alpha, \beta, \nu, k) m_{\nu}(x)
$$

where $d(\alpha, \beta, \nu, k)$ are certain constants and the coefficient of $m_{\lambda}$ is as above. Applying the transformation $x_{i}=\frac{1}{2}-\frac{1}{4}\left(e^{t_{i}}+e^{-t_{i}}\right)$ will result in a $W_{B}$-invariant polynomial on $H_{B}$ which has as support the $W_{B}$-orbit of the elements in $\Pi_{B}^{+}(\lambda)$, which is $\Pi_{B}(\lambda)$. Hence $G_{\lambda}^{(\alpha, \beta)}$ is a $W_{B}$-invariant polynomial of the form $\sum_{\nu \in \Pi_{B}(\lambda)} d^{\prime}(\alpha, \beta, \nu, k) e^{\nu}$ for certain constants $d^{\prime}(\alpha, \beta, \nu, k)$. We have seen above that $G_{\lambda}^{(\alpha, \beta)}$ is an eigenfunction of $L_{B}(\kappa)$ with eigenvalue $\left\langle\lambda, \lambda+2 \rho_{B}(\kappa)\right\rangle$ where $\kappa=\left(k_{1}, k_{2}, k_{3}\right)=\left(\alpha-\beta, \beta+\frac{1}{2}, k\right)$. As in Proposition 4.1 these two facts allows one to conclude that the $G_{\lambda}^{(\alpha, \beta)}$ are exactly the Jacobi polynomials $P_{B}(\lambda, \kappa)$, up to normalization. Now the coefficient of $e^{\lambda}$ in $G_{\lambda}^{(\alpha, \beta)}$ will equal

$$
\left(-\frac{1}{4}\right)^{|\lambda|} \frac{u_{\lambda \lambda} v_{\lambda \lambda}\left(k^{-1}\right)}{J_{\lambda}\left(1_{n} ; k^{-1}\right)}=\frac{2^{-2|\lambda|} h_{*}(\lambda)}{J_{\lambda}\left(1_{n} ; k^{-1}\right)} .
$$

Here the factor $\left(-\frac{1}{4}\right)^{|\lambda|}$ enters because of the coordinate change. Since the coefficient of $e^{\lambda}$ in $P_{B}(\lambda, \kappa)$ is 1 , the theorem follows.

For $\lambda=\left(l^{n}\right)=l \omega_{n} \in P_{B}^{+}$we can combine (4.10) and Theorem 5.1 to obtain that with $\kappa=\left(k_{1}, k_{2}, k_{3}\right)=\left(\alpha-\beta, \beta+\frac{1}{2}, k\right)$ one has

$$
G_{l \omega_{n}}^{(\alpha, \beta)}\left(x ; k^{-1}\right)=\frac{2^{-2 n l} h_{*}\left(l \omega_{n}\right)}{J_{l \omega_{n}}\left(1_{n} ; k^{-1}\right) \mathbf{c}_{B}\left(l \omega_{n}+\rho_{B}(\kappa), \kappa\right)}{ }_{2} F_{1}\left(-l, b ; c ; x ; k^{-1}\right)
$$

where $b=\alpha+\beta+1+k(n-1)+l$ and $c=\alpha+1+k(n-1)$. From (3.12) we have $h_{*}\left(l \omega_{n}\right)=J_{l \omega_{n}}\left(1_{n} ; k^{-1}\right)$ while in (4.15) we have shown that $\mathbf{c}_{B}\left(l \omega_{n}+\rho_{B}(\kappa), \kappa\right)=2^{-2 n l} b_{\left(l^{n}\right)} /(c)_{\left(l^{n}\right)}$. Hence we have obtained the following result. 
Corollary 5.2. One has

$$
G_{\left(l^{n}\right)}^{(\alpha, \beta)}\left(x ; k^{-1}\right)=\frac{(c)_{\left(l^{n}\right)}}{(b)_{\left(l^{n}\right)}} F_{1}\left(-l, b ; c ; x ; k^{-1}\right)
$$

where $b=\alpha+\beta+2+k(n-1)+l$ and $c=\alpha+k(n-1)+1$.

The first result in this direction was obtained by Koornwinder in the two variable case and with $\gamma=0$ (cf. [14, (4.13)]). Using a different method Corollary 5.2 has also been obtained very recently by Kaneko [13, Theorem 5].

Corollary 5.3. In ${ }_{2} F_{1}\left(-l, b ; c ; x ; k^{-1}\right)$ the coefficient of $\Omega_{\left(l^{n}\right)}\left(x ; k^{-1}\right)$ equals

$$
\frac{(-l)_{\lambda}(b)_{\lambda}}{(c)_{\lambda} k^{|\lambda|}} \frac{J_{\lambda}\left(1_{n} ; k^{-1}\right)}{\left\langle J_{\lambda}, J_{\lambda}\right\rangle_{k}}=(-1)^{|\lambda|} \frac{(b)_{\lambda}}{(c)_{\lambda}}, \quad \lambda=l \omega_{n}=\left(l^{n}\right) .
$$

Proof. In general the coefficient of $\Omega_{\lambda}$ in $G_{\lambda}^{(\alpha, \beta)}$ is $(-1)^{|\lambda|}$ while for $\lambda=l \omega_{n}$ we can read off the coefficient of $\Omega_{l \omega_{n}}$ in ${ }_{2} F_{1}\left(-l, b ; c ; x ; k^{-1}\right)$ from (4.7). Thus Corollary 5.3 follows immediately from Corollary 5.2. Based on (3.12), (3.17) and the definition of $h^{*}(\lambda)$ in (3.7) it is easy to give a direct proof of (5.10). Using (3.16) one can also obtain (5.10) as the special case $\lambda=\left(l^{n}\right)$ of (3.18) (note that $\pi\left(l^{n}\right)=0$ ).

Corollary 5.2 gives explicit expressions for the coefficients $u_{\lambda \mu}(\alpha, \beta, k)$ of $\Omega_{\mu}$ in the expansion (5.3) of $G_{\left(l^{n}\right)}^{(\alpha, \beta)}$. In fact

$$
u_{\left(l^{n}\right), \mu}(\alpha, \beta, k)=(-l)_{\mu} \frac{(b)_{\mu}(c)_{\left(l^{n}\right)}}{(b)_{\left(l^{n}\right)}(c)_{\mu}} \frac{J_{\mu}\left(1_{n} ; k^{-1}\right)}{\left\langle J_{\mu}, J_{\mu}\right\rangle_{k} k^{|\mu|}}, \quad \mu \subset\left(l^{n}\right),
$$

where $J_{\mu}\left(1_{n} ; k^{-1}\right)$ and $\left\langle J_{\mu}, J_{\mu}\right\rangle_{k}$ are known (see (3.13) and (3.17)), $c=\alpha+$ $k(n-1)+1$ and $b=\alpha+\beta+k(n-1)+1+l$. For general $\lambda$ Macdonald [24, $\S 9]$ writes

$$
G_{\lambda}^{(\alpha, \beta)}=\sum_{\mu \subset \lambda}(-1)^{|\mu|} \frac{(\alpha+1+k(n-1))_{\lambda}}{(\alpha+1+k(n-1))_{\mu}} c_{\lambda / \mu}(\alpha+\beta+2+2 k(n-1)) \Omega_{\mu}
$$

for certain coefficients $c_{\lambda / \mu}$. Expansions like this were first studied extensively by Koornwinder and Sprinkhuizen-Kuyper in [14] for the two variable case. As for the case $k=\frac{1}{2}$ in [10] Macdonald obtains from the differential equation (5.4) a recurrence relation for these coefficients. Solving this recurrence relation then leads to the following expression for these coefficients $c_{\lambda / \mu}$ :

$$
c_{\lambda / \mu}(C)=\sum_{T} \prod_{i=1}^{r}\left(\begin{array}{c}
\lambda^{(i-1)} \\
\lambda^{(i)}
\end{array}\right) /\left(i C+2 \rho(\lambda)-2 \rho\left(\lambda^{(i)}\right)\right)
$$

where $C=\alpha+\beta+2+2 k(n-1), \rho(\lambda)=n\left(\lambda^{\prime}\right)-k n(\lambda)$ and the sum is over all standard tableaux $\lambda=\lambda^{(0)} \supset \lambda^{(1)} \supset \cdots \supset \lambda^{(r)}=\mu$ of shape $\lambda / \mu$. In particular $|\lambda|-|\mu|=r$ and $\left|\lambda^{(i)}\right|-\left|\lambda^{(i-1)}\right|=1$. For a definition of the generalized binomial coefficients $\left(\begin{array}{l}\lambda \\ \mu\end{array}\right)$ for partitions $\mu \subset \lambda$ see, e.g., $[24, \S 6,32$, $\S 2$ and 17, §3]. Although the partitions which occur here are known from the work of Lassalle [17], the resulting sum over all standard tableaux of shape $\lambda / \mu$ seems hard to evaluate. For the case $\lambda=\left(l^{n}\right)$ we obtain from (5.11) a simple explicit expression for $c_{\left(l^{n}\right) / \mu}(C)$. 
Corollary 5.4. Let $c_{\lambda / \mu}$ be defined by (5.12). Then

$$
\begin{aligned}
& \frac{(b)_{\left.()^{n}\right)}}{(b)_{\mu}} c_{\left(l^{n}\right) / \mu}(\alpha+\beta+2+2 k(n-1)) \\
& \quad=(-1)^{|\mu|}(-l)_{\mu} \frac{J_{\mu}\left(1_{n} ; k^{-1}\right)}{\left\langle J_{\mu}, J_{\mu}\right\rangle_{k} k^{|\mu|}}, \quad \mu \subset\left(l^{n}\right),
\end{aligned}
$$

where $b=\alpha+\beta+1+k(n-1)+l, c=\alpha+k(n-1)+1$ and $J_{\mu}\left(1_{n} ; k^{-1}\right)$ and $\left\langle J_{\mu}, J_{\mu}\right\rangle_{k}$ are given by (3.13) and (3.17).

Note that the right-hand side of (5.14) does not depend on $\alpha$ and $\beta$. Let us consider some special cases. First we take $l=1$, so that $\mu \subset\left(1^{n}\right)$ is the set $\left\{\left(1^{s}\right) \mid s=0,1, \ldots, n\right\}$. From (3.13) and (3.17) follows that $J_{\left(1^{s}\right)}\left(1_{n} ; k^{-1}\right)=$ $\left(\begin{array}{l}n \\ s\end{array}\right) s !$ and $k^{s}\left\langle J_{\left(1^{s}\right)}, J_{\left(1^{s}\right)}\right\rangle_{k}=s !(k(s-1)+1)_{\left(1^{s}\right)}$. An easy calculation shows that in general $(-l)_{\left(l^{n}\right)}=(-1)^{n l}(k(n-1)+1)_{\left(l^{n}\right)}$. Hence we have

$$
(-1)^{|\mu|}(-1)_{\mu} \frac{J_{\mu}\left(1_{n} ; k^{-1}\right)}{\left\langle J_{\mu}, J_{\mu}\right\rangle_{k} k^{|\mu|}}=\left(\begin{array}{l}
n \\
s
\end{array}\right), \quad \mu=\left(1^{s}\right),
$$

and so the expression for $u_{\left(1^{n}\right),\left(1^{s}\right)}$ in (5.11) reduces to

$$
u_{\left(1^{n}\right),\left(1^{s}\right)}=(-1)^{s}\left(\begin{array}{l}
n \\
s
\end{array}\right) \frac{(b)_{\left(1^{s}\right)}(c)_{\left(1^{n}\right)}}{(b)_{\left(1^{n}\right)}(c)_{\left(1^{s}\right)}}
$$

It follows that

$$
G_{\left(1^{n}\right)}^{(\alpha, \beta)}\left(x ; k^{-1}\right)=\frac{(c)_{\left(1^{n}\right)}}{(b)_{\left(1^{n}\right)}} \sum_{s=0}^{n}(-1)^{s}\left(\begin{array}{l}
n \\
s
\end{array}\right) \frac{(b)_{\left(1^{s}\right)}}{(c)_{\left(1^{s}\right)}} \Omega_{\left(1^{s}\right)}\left(x ; k^{-1}\right),
$$

a result which was previously obtained by Macdonald [24, $\left.(9.21)^{\prime}\right]$. In fact Macdonald gives the expansion even for $G_{\left(1^{r}\right)}^{(\alpha, \beta)}\left(x ; k^{-1}\right)$ with $0 \leq r \leq n$. In order to compare (5.16) with $\left[24,(9.21)^{\prime}\right]$ we note that for $n \geq s$ we have

$$
\frac{(b)_{\left(1^{n}\right)}}{(b)_{\left(1^{s}\right)}}=\prod_{i=1}^{n-s}(b+k(n-1)-k(n+s+i-2))
$$

and that for general $l$ our pairs $(c, b)$ and $(\alpha, \beta)$ correspond to $(A, C-$ $k(n-1)-1+l)$ and $(a, b)$ respectively in $[24, \S 9]$.

Another special case we want to mention is $\mu=\left(l^{n-1}, l-r\right)$ with $r \in$ $\{0,1, \ldots, l\}$ so that in particular $|\lambda|-|\mu|=r$. We omit the details of the calculation. The results are as follows.

$$
(-1)^{|\mu|}(-l)_{\mu} \frac{J_{\mu}\left(1_{n} ; k^{-1}\right)}{\left\langle J_{\mu}, J_{\mu}\right\rangle_{k} k^{|\mu|}}=\left(\begin{array}{l}
l \\
r
\end{array}\right) \frac{(k n)_{r}}{(k)_{r}}, \quad \mu=\left(l^{n-1}, l-r\right) .
$$

Note that the identity (5.10) is the special case $r=0$ of (5.17). Also note the relation with (3.18). If $\lambda=\left(l^{n}\right)$ and $\mu=\left(l^{n-1}, l-r\right)$ then there is only one standard tableaux of shape $\lambda / \mu$, namely the tableaux $\lambda=\lambda^{(0)} \supset \lambda^{(1)} \supset \cdots \supset$ $\lambda^{(r)}=\mu$ with $\lambda^{(i)}=\left(l^{n-1}, l-i\right)$. Hence the sum in (5.13) reduces to one term only. Now it is not very hard to show that with $\lambda$ and $\mu$ as above one has

$$
\prod_{i=1}^{r}\left(i C+2 \rho(\lambda)-2 \rho\left(\lambda^{(i)}\right)\right)=r ! \frac{(b)_{(l n)}}{(b)_{\mu}}
$$


so that

$$
r ! \frac{(b)_{\left(l^{n}\right)}}{(b)_{\mu}} c_{\left(l^{n}\right) / \mu}=\prod_{i=1}^{r}\left(\begin{array}{c}
\lambda^{(i-1)} \\
\lambda^{(i)}
\end{array}\right) .
$$

Hence it follows from (5.14) that $(5.17)$ is equivalent to

$$
\prod_{i=1}^{r}\left(\begin{array}{c}
\lambda^{(i-1)} \\
\lambda^{(i)}
\end{array}\right)=\frac{l !}{(l-r) !} \frac{(k n)_{r}}{(k)_{r}}
$$

for $r=1,2, \ldots, l$. From (5.18) follows easily that for $i=1,2, \ldots, l$

$$
\left(\begin{array}{c}
\lambda^{(i)} \\
\lambda^{(i+1)}
\end{array}\right)=(k-i) \frac{k n+i}{k+i},
$$

a result which also follows immediately from the much more general results obtained by Lassalle in [17, $\S 3]$. For the case $k=\frac{1}{2}$ the binomial coefficients for partitions were introduced by Constantine. For references and more details concerning the case $k=\frac{1}{2}$ we refer to [26, §7.5] (also see e.g. [10]) while for $k$ arbitrary we refer to [24, 33 and especially 17 and 18].

\section{ACKNOWLEDGMENT}

The first-named author carried out the major part of the research for this paper while he held a visiting position at the University of Texas at Austin, Austin, Texas. He would like to thank the University of Texas at Austin for its hospitality and especially John Gilbert for his stimulating discussions on the hypergeometric function of matrix argument.

\section{REFERENCES}

1. R. J. Beerends, Chebyshev polynomials in several variables and the radial part of the LaplaceBeltrami operator, Trans. Amer. Math. Soc. 328 (1991), 779-814.

2. A. G. Constantine, Some non-central distribution problems in multivariate analysis, Ann. Math. Statist. 34 (1963), 1270-1285.

3. A. Debiard, Système différentiel hypergéométrique et parties radiales des opérateurs invariants des espaces symétriques de type $B C_{p}$, Séminaire d'algèbre Paul Dubreil et Marie-Paule Malliavin, Lecture Notes in Math., vol. 1296, Springer, Berlin, 1987.

4. J. Faraut and A. Korányi, Fonctions hypergéométrique associées aux cônes symétriques, C. R. Acad. Sci. Paris Sér. I 307 (1988), 555-558.

5. K. I. Gross and D. St. P. Richards, Special functions of matrix argument. I: Algebraic induction, zonal polynomials, and hypergeometric functions, Trans. Amer. Math. Soc. 301 (1987), 781-811.

6. G. J. Heckman and E. M. Opdam, Root systems and hypergeometric functions. I, Compositio Math. 64 (1987), 329-352.

7. G. J. Heckman, Root systems and hypergeometric functions. II, Compositio Math. 64 (1987), 353-374.

8. C. S. Herz, Bessel functions of matrix argument, Ann. of Math. (2) 61 (1955), 474-523.

9. A. T. James, Distributions of matrix variates and latent roots derived from normal samples, Ann. Math. Statist. 35 (1964), 475-501.

10. A. T. James and A. G. Constantine, Generalized Jacobi polynomials as spherical functions of the Grassmann manifold, Proc. London Math. Soc. 29 (1974), 174-192.

11. K. W. J. Kadell, $A$ proof of some q-analogues of Selberg's integral for $k=1$, SIAM J. Math. Anal. 19 (1988), 944-968. 
12. _ The Selberg-Jack symmetric function, preprint 1988.

13. J. Kaneko, Selberg integrals and hypergeometric functions associated with Jack polynomials, preprint, Dept. of Math., Kyushu Univ., Fukuoka, Japan.

14. T. H. Koornwinder and I. G. Sprinkhuizen-Kuyper, Generalized power series expansions for a class of orthogonal polynomials in two variables, SIAM J. Math. Anal. 9 (1978), 457-483.

15. A. Korányi, Hua-type integrals, hypergeometric functions and symmetric polynomials, International symposium in memory of Hua Loo Keng, vol. II, Analysis, (S. Gong et al., eds.), Science Press, Beijing and Springer-Verlag, Berlin, 1991, pp. 169-180.

16. _ Transformation properties of the generalized Muirhead operators, preprint 1990.

17. M. Lassalle, Une formule du binôme généralisée pour les polynômes de Jack, C. R. Acad. Sci. Paris Sér. I 310 (1990), 253-256.

18. __ Coefficients du binôme généralisés, C. R. Acad. Sci. Paris Sér. I 310 (1990), 257-260.

19. I. G. Macdonald, Symmetric functions and Hall polynomials, Oxford Univ. Press, Oxford, 1979.

20. __ Symmetric functions and Hall polynomials, 2nd ed., Oxford Univ. Press, Oxford (to appear).

21. __ Commuting differential operators and zonal spherical functions, Algebraic Groups (Utrecht, 1986), (A. M. Cohen et al., eds.), Lecture Notes in Math., vol. 1271, SpringerVerlag, Berlin, 1987.

22. __ A new class of symmetric functions, Publ. IRMA Strasbourg, Séminaire Lotharingien, 1988, pp. $131-171$.

23. __ Orthogonal polynomials associated with roots systems, Orthogonal Polynomials: Theory and Practice (P. Nevai, ed.), NATO ASI Series C, vol. 294, Kluwer, Dordrecht, 1990.

24. __ Hypergeometric functions, unpublished manuscript.

25. R. J. Muirhead, Systems of partial differential equations for hypergeometric functions of matrix argument, Ann. Math. Statist. 41 (1970), 991-1001.

26. __ Aspects of multivariate statistical theory, Wiley, New York, 1982.

27. E. M. Opdam, Root systems and hypergeometric functions. III, Compositio Math. 67 (1988), 21-49.

28. __ Some applications of hypergeometric shift operators, Invent. Math. 98 (1989), 1-18.

29. __ An analogue of the Gauss summation formula for hypergeometric functions related to root systems, Math. Z. 212 (1993), 313-336.

30. R. P. Stanley, Some combinatorial properties of Jack symmetric functions, Adv. in Math. 77 (1989), 76-115.

31. L. Vretare, Formulas for elementary spherical functions and generalized Jacobi polynomials, SIAM J. Math. Anal. 15 (1984), 805-833.

32. Z. Yan, Generalized hypergeometric functions, C. R. Acad. Sci. Paris Sér. I 310 (1990), 349-354.

33. __ A class of generalized hypergeometric functions, Thesis, CUNY, 1990.

Department of Mathematics, University of Leiden, P. O. Box 9512, 2300 RA Leiden, THE NETHERLANDS

Current address, R. J. Beerends: Department TW, Open University, P. O. Box 2960, 6401 DL Heerlen, The Netherlands

E-mail address, E. M. Opdam: opdam@rulcri.leidenuniv.nl 